\title{
Numerical investigations of foam-like materials by nested high-order finite element methods
}

\author{
H. -G. Sehlhorst • R. Jänicke • A. Düster · E. Rank • \\ H. Steeb · S. Diebels
}

Received: 19 December 2008 / Accepted: 20 August 2009 / Published online: 18 September 2009

(C) The Author(s) 2009. This article is published with open access at Springerlink.com

\begin{abstract}
In this paper we present a multiscale framework suited for geometrically nonlinear computations of foam-like materials applying high-order finite elements ( $p$-FEM). This framework is based on a nested finite element analysis (FEA) on two scales, one nonlinear boundary value problem on the macroscale and $k$ independent nonlinear boundary value problems on the microscale allowing for distributed computing. The two scales are coupled by a numerical projection and homogenization procedure. On the microscale the foamlike structures are discretized by high-order continuum-based finite elements, which are known to be very efficient and robust with respect to locking effects. In our numerical examples we will discuss in detail three characteristic test cases (simple shear, tension and bending). Special emphasis is placed on the material's deformation-induced anisotropy and the macroscopic load-displacement behavior.
\end{abstract}

H.-G. Sehlhorst $(\varangle) \cdot$ A. Düster

Numerische Strukturanalyse mit Anwendungen in der

Schiffstechnik, Schwarzenbergstrasse 95 c,

Technische Universität Hamburg-Harburg,

21073 Hamburg, Germany

e-mail: hans-georg.sehlhorst@tu-harburg.de

R. Jänicke $\cdot S$. Diebels

Lehrstuhl für Technische Mechanik, Universität des Saarlandes, 66123 Saarbrücken, Germany

E. Rank

Lehrstuhl für Computation in Engineering, Technische Universität

München, Arcisstr. 21, 80290 München, Germany

H. Steeb

Lehrstuhl für Kontinuumsmechanik, Ruhr-Universität Bochum,

Universitätsstr. 150, 44780 Bochum, Germany

H. Steeb

Multi Scale Mechanics, TS, CTW, UTwente,

P.O. Box 217, 7500 AE Enschede, The Netherlands
Keywords Cellular foams · Homogenization . Large deformations

\section{Introduction}

There is an increasing interest in the application of foam-like materials in science and industry. However, these materials show a complex mechanical behavior due to size effects, refer to e.g. Onck [34], and deformation-induced anisotropy, refer to e.g. Hohe and Becker [24], under large displacements. In this paper the latter phenomenon will be investigated, size effects are not considered but will be addressed in future work.

This section is structured as follows: We start with an overview of numerical modeling for foam-like materials. Afterwards, important publications concerning large deformations of foam-like materials are summarized and finally we motivate and describe our approach.

There are basically two different approaches for numerical modeling of open-cell foam-like materials: The microscopic approach, see e.g. Diebels and Steeb [3], Gibson and Ashby [18], Tekoğlu [40] and the macroscopic approach, see e.g. Eringen et al. [13,14,25], Lakes [1], Diebels and Steeb [4]. In the first approach, the structure is microscopically resolved (e.g. by beam elements) while in the second one a phenomenological formulation is applied. In the latter approach the structure is macroscopically discretized by finite elements based on extended continuum theories (i.e. micropolar theories), refer to e.g. Lakes [1], Ebinger et al. [12], Onck [34]. These theories can capture higher-order effects (e.g. size effects). Although the numerical efficiency is much higher, this macroscopic approach is limited by the fact that the material parameters are difficult to obtain as shown by Lakes [28]. 
A combination of both approaches is called the $\mathrm{FE}^{2}$ method where a microstructure, either a representative volume element (RVE) or a testing volume element (TVE) as introduced by Nemat-Nasser and Hori [31], is embedded into a macroscopic finite element framework via projection and homogenization schemes, refer to Feyel [15-17], Kouznetsova [27]. The RVE is chosen such that it represents the micromechanical properties of the material. In general this leads to large RVEs and therefore to a high number of degrees of freedom, as demonstrated for hardened cement paste by Hain [19]. In contrast, the TVE is much smaller than the RVE. The TVE only provides the basic mechanical properties of the material, i.e. it can be too stiff or too weak depending on its boundary conditions. There are firstorder $\mathrm{FE}^{2}$ schemes with standard continuum theories on both macro- and microlevel, and schemes of higher-order with extended continuum theories applied to the macrolevel.

With respect to the present paper it is worth to highlight publications concerning nonlinear analysis of foam-like materials: Zhu et al. [45] subjected tetrakaidecahedral cells to high strain compression, and the numerical results were validated experimentally. Similar investigations can be found in works of e.g. Laroussi et al. [29] or Wang and Cuitiño [42]. Note, that Wang and Cuitiño included the analysis of deformation-induced anisotropy and anisotropy depending on the initial cell orientation.

Two-dimensional computations with honeycomb structures under large deformations including plasticity can be found in Triantafyllidis and Schraad [41]. Ohno et al. [33] presented a homogenization framework allowing for studying microscopic bifurcation under compression. A method to determine an appropriate number of unit cells in an RVE was described by Saiki et al. [35]. Different load cases (tension, compression, and shear) were studied within a homogenization concept proposed by Hohe and Becker [24]. Here, deformation-induced anisotropy was detected, too.

In the above mentioned publications the microstructures (i.e. the cell walls) were discretized by either dimensionally reduced elements (i.e. beam elements) or by low-order continuum elements. The authors of this contribution have propagated this discretization strategy (and with restriction to small displacements) in previous works $[3,11,12]$, too. In contrast, the present paper uses continuum finite elements of high-order to discretize the cell walls within a two-dimensional hyperelastic $\mathrm{FE}^{2}$ framework of first-order. This novel approach in multiscale modeling has several advantages:

- High-order elements have been demonstrated to provide high convergence rates and robustness against locking effects in linear and nonlinear problems of solid/structural mechanics as shown by the authors et al. [6-10,21, $32,38]$.
- In a continuum-based approach the implementation of geometrical nonlinearities is straight-forward since no drilling degrees of freedom arising in classical beam, plate, and shell theories need to be considered.

- Thin- and thick-walled beam-like structures can be accurately discretized with anisotropic continuum-based elements, where different polynomial degrees are chosen in longitudinal and thickness direction.

In the numerical examples we discuss a suitable discretization of the cell walls with our high-order finite elements. Then we apply our framework to uniaxial tension, simple shear, and bending tests. The choice of different boundary conditions for the microstructures is discussed and it is demonstrated that the proposed framework is able to capture the deformation-induced anisotropy. Additionally, the characteristic load-displacement behavior in the different tests is investigated.

\section{The FE $^{2}$ approach}

In this section we review the $\mathrm{FE}^{2}$ method of first-order for the geometrically nonlinear case as presented in Feyel and Chaboche [17] and Kouznetsova [27]. In the following, index $M$ indicates macroscopic quantities and $m$ is used for microscopic quantities. The method is based on the numerical solution of nested boundary value problems (BVPs) where one has to solve a macroscopic nonlinear $\mathrm{BVP}_{M}$ and $k$ independent nonlinear $\mathrm{BVP}_{m}$ on the microscale. Scale separation is assumed allowing to treat the problems on the microscale independently. The main idea for coupling both scales is expressed as follows: At each macroscopic integration point the gradient of the displacement field w.r.t. the reference configuration (i.e. $\operatorname{Gradu}_{M}$ ) is projected onto the microstructure and homogenized Cauchy stresses $\sigma_{M}$ are transferred back after solving the microscopic BVP, cf. Fig. 1.

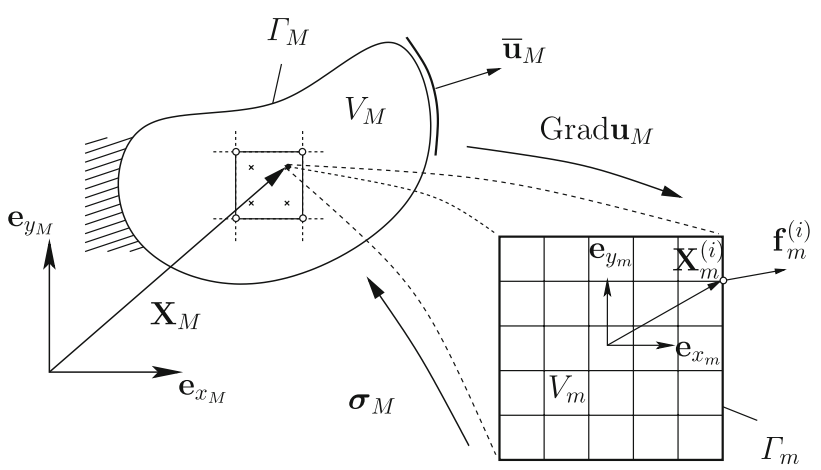

Fig. 1 Nested boundary value problem in the reference configuration 
To be more specific, let us consider the underlying formulation. By the projection of $\operatorname{Gradu}_{M}$

$\overline{\mathbf{u}}_{m}=\operatorname{Grad} \mathbf{u}_{M} \cdot \mathbf{X}_{m}$

onto the microstructure's boundary $\Gamma_{m}$ in its reference configuration the microscopic Dirichlet $\mathrm{BVP}_{m}$ is defined. Here, $\mathbf{X}_{m}$ is the so-called branch vector of the reference configuration related to the center of the microstructure, refer to Fig. 1. The homogenization is based on an averaging theorem as stated in $[27,31]$ and where

$\langle *\rangle=\frac{1}{V_{m}} \int_{V_{m}}(*) d V_{m}$.

In (2) $V_{m}$ represents the volume of the microstructure. The application of (2) leads to the first Piola-Kirchhoff stress tensor

$$
\left\langle\mathbf{P}_{M}\right\rangle=\frac{1}{V_{m}} \int_{\Gamma_{m}} \mathbf{p}_{m} \otimes \mathbf{X}_{m} d \Gamma_{m} .
$$

In Eq. (3) the integral of the dyadic product of the first PiolaKirchhoff stress vector $\mathbf{p}_{m}=\mathbf{P}_{m} \cdot \mathbf{N}_{m}$ and the branch vector over the boundary $\Gamma_{m}$ is computed assuming unit thickness (where $\mathbf{P}_{m}$ is the first Piola-Kirchhoff stress tensor, and $\mathbf{N}_{m}$ is the outer normal vector defined on $\Gamma_{m}$ ). The integration can be simplified when using discrete quantities at the nodes of the finite element discretization of the microscopic structure:

$\left\langle\mathbf{P}_{M}\right\rangle=\frac{1}{V_{m}} \sum_{i=1}^{n} \mathbf{f}_{m}^{(i)} \otimes \mathbf{X}_{m}^{(i)}$

To this end the sum of the dyadic product of the nodal forces $\mathbf{f}_{m}^{(i)}$ and the branch vector for all boundary nodes $(i=$ $1, \ldots, n)$ is evaluated. In order to obtain the Cauchy stresses from (4) we perform a push forward

$\boldsymbol{\sigma}_{M}=\frac{1}{J_{M}}\left\langle\mathbf{P}_{M}\right\rangle \cdot \mathbf{F}_{M}$

using the deformation gradient $\mathbf{F}_{M}=\operatorname{Gradu}_{M}+\mathbf{I}$ and its determinant $J_{M}=\operatorname{det}\left(\mathbf{F}_{M}\right)$. It is important to mention, that Eq. (2) cannot be applied to Cauchy stresses since

$\sigma_{M} \neq\left\langle\sigma_{m}\right\rangle$

in general according to the nonlinearity of (5), see Hill [23] or Nemat-Nasser and Hori [31].

Next, implementation aspects are presented. Since we have $k$ independent microscopic (geometrically nonlinear) $\mathrm{BVP}_{m} \mathrm{~s}$ the $\mathrm{FE}^{2}$ approach is perfectly parallelizable. This is illustrated in Fig. 2.

Within the nonlinear $\mathrm{BVP}_{M}$ the Master program distributes in each step of the iterative solution procedure the $k$ independent $\mathrm{BVP}_{m} \mathrm{~s}$ to the Workers, obtains the homogenized stresses $\sigma_{M}$ and solves the macroscopic equation

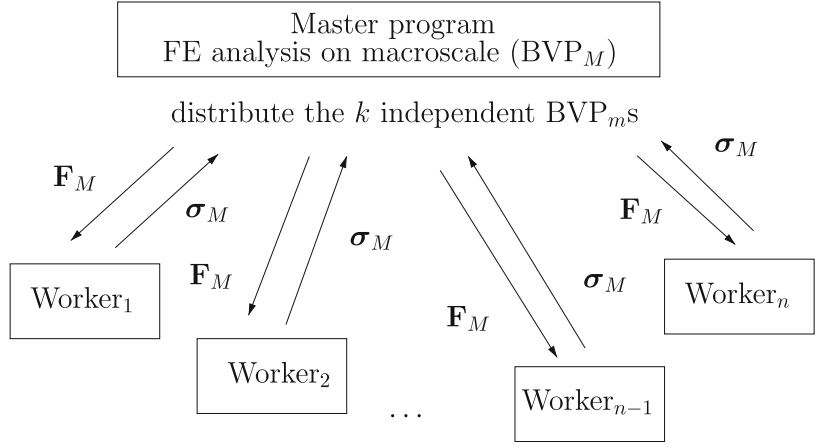

Fig. 2 Distributed computing of microscopic $\mathrm{BVP}_{m} \mathrm{~s}$

system. The Master program is based on a discrete NewtonRaphson algorithm as described in Wriggers [43] where the tangential stiffness matrix is computed by numerical differentiation of the finite element residual. Note, that this numerical differentiation procedure is sufficient since we are only dealing with geometric nonlinearities, for including material nonlinearities like viscosity or damage suitable algorithms are described in works of Miehe, e.g. [30]. On the microlevel (i.e. the Workers) a hyperelastic high-order finite element formulation as described in Sect. 3 is applied. The different $\mathrm{BVP}_{m} \mathrm{~s}$ are solved by a Newton-Raphson scheme on a Linux cluster. The Master process runs on an AMD Athlon 2500+ CPU whereas the 10 Worker nodes are based on Intel 2.4 $\mathrm{GHz}$ CPUs.

\section{High-order finite elements for hyperelastic problems}

In the following we present the basic concept of our hyperelastic high-order finite element formulation. In a first step the hyperelastic material model applied in this paper is presented. Afterwards the anisotropic beam-like continuum finite element formulation of high-order designed for discretizing thin-walled microstructures is briefly summarized. Finally, we numerically demonstrate some important advantages of our high-order finite elements addressing the convergence rate and the capability to discretize thin- as well as thick-walled structures.

\subsection{Hyperelasticity}

Materials, for which a constitutive behavior is only a function of the current state of deformation, are called elastic [2]. A Neo-Hookean material is an example for this class of materials. The strain energy (density) function per unit volume is given by

$\Psi=\frac{\mu}{2}\left(I_{C}-3\right)-\mu \ln J+\frac{\Lambda}{2}(\ln J)^{2}$ 


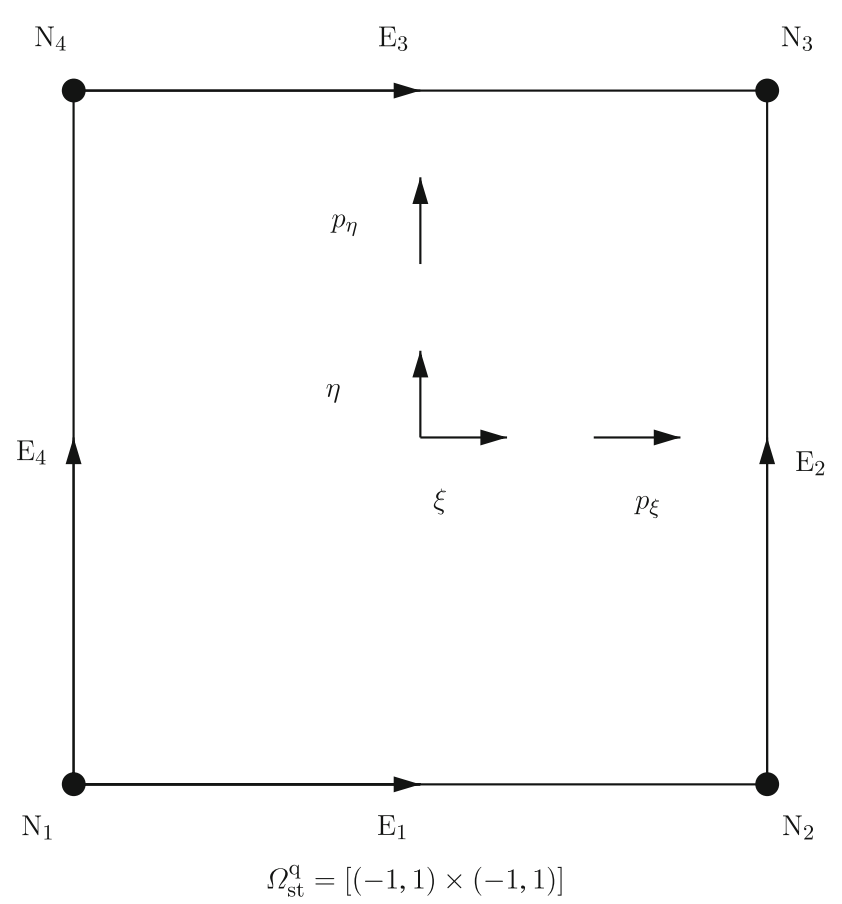

Fig. 3 Standard quadrilateral element: Definition of nodes, edges and polynomial degrees

where $I_{C}$ is the first invariant of the right Cauchy-Green tensor $\mathbf{C}=\mathbf{F}^{T} \cdot \mathbf{F}, J$ is the determinant of the deformation gradient $\mathbf{F}$, and $\Lambda$ and $\mu$ are material constants.

\subsection{A high-order beam-like solid finite element formulation}

Our beam-like, high-order solid finite element formulation is based on a quadrilateral element, applying the shape functions proposed by Szabó and Babuška [37].

The formulation allows the polynomial degree to be varied for the two different local directions $(\xi, \eta)$, see Fig. 3.

For efficiency reasons it is important to treat the thickness direction differently, when thin-walled structures are to be discretized. In this paper this is accounted for by using anisotropic Ansatz functions for the two-dimensional displacement field. Therefore, the polynomial degree $q_{e}$ in the thickness direction is chosen differently from the polynomial degree $p_{e}$ in the longitudinal direction, see Fig. 4.

Since the $p$-version is less prone to locking effects related to thin-walled structures $[6,38]$ or incompressible materials $[7,22]$, a pure displacement based formulation can be applied. In order to solve the nonlinear problem, an incremental/iterative procedure based on the Newton-Raphson scheme is applied. Thanks to the spatial formulation applied in this work, the strain displacement matrix is not fully populated (as compared to a material formulation). Taking advantage of the sparsity of the strain displacement matrix the

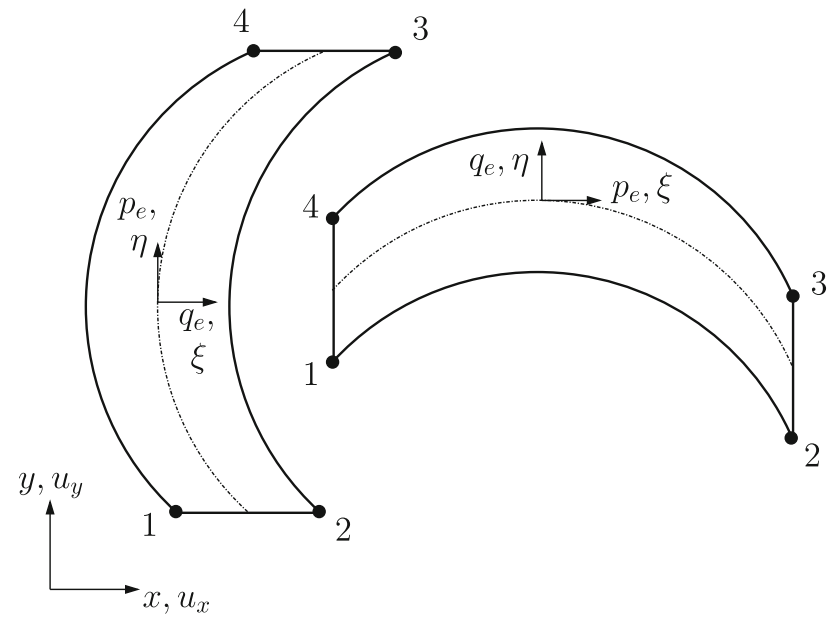

Fig. 4 Beam-like element with different local orientations and polynomial degrees

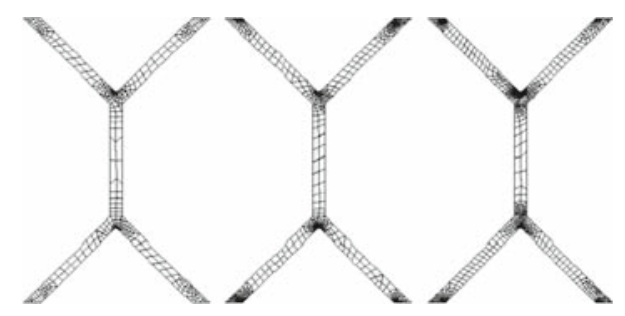

Fig. $5 h$-adaptive refinement with 366, 650, 1036 quadrilaterals $(p=2)$

integration of the element stiffness matrices can be significantly accelerated, see for example [20,43].

\subsection{Preliminary study}

The performance of our $p$-elements w.r.t. convergence rates and wall-thickness effects is demonstrated in the following preliminary study. Here, only linear elasticity (small deformations) is considered for simplicity. Note however, that the basic properties of the $p$-version (high convergence rates, robustness against locking) are valid for both linear and nonlinear problems $[7,21,38]$.

In our preliminary study, a beam-like structure corresponding to a TVE of a foam-like material is investigated. Two discretization strategies based on two-dimensional continuum elements are compared: Fig. 5 shows the meshes for an adaptive $h$-refinement $(p=2)$, and Fig. 6 shows the $p$-version's mesh. The $h$-version's meshes are obtained by an adaptive refinement, driven by a residual based error estimator, see [5], whereas the $p$-refinement is based on a fixed mesh resolving singularities at the boundary.

We consider the load case as shown in Fig. 7 where Dirichlet boundary conditions are applied resulting in the sketched deformation pattern. Firstly, we focus on the convergence rates: To this end, the reaction forces/moments 


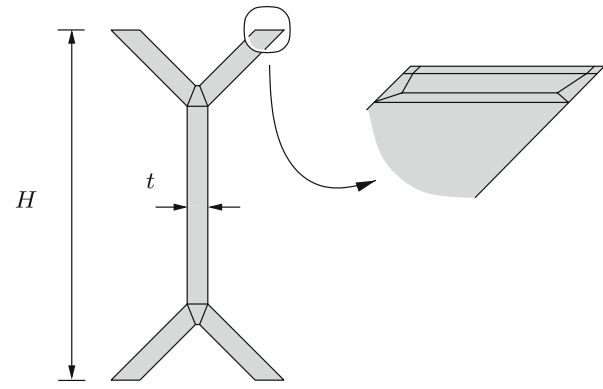

Fig. 6 -version mesh with 35 quadrilaterals, including boundary refinement and dimensioning

Fig. 7 Load case with Dirichlet boundary conditions, scaling factor $=1.28$
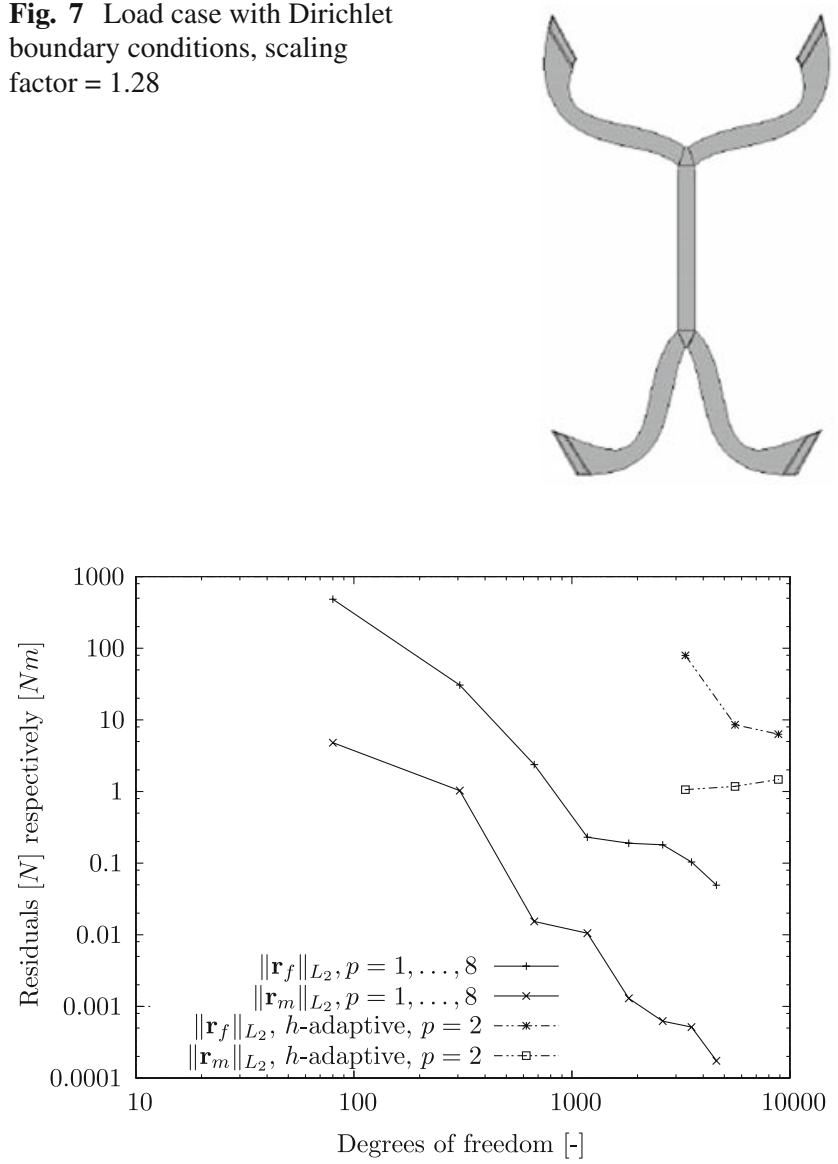

Fig. 8 Residuals of forces/moments for uniform $p$-/ adaptive $h$-refinement; note that the finest resolution in both approaches (i.e. $p=8$ respectively the finest $h$-mesh with 1036 quadrilaterals) requires a computation time of $1 \mathrm{~s}$

at the Dirichlet boundaries are computed by performing an integration of the stresses. Since the reaction forces/moments depend on the discretization, we can introduce residuals for the equilibrium of forces/moments. These residuals are plotted in Fig. 8. One can clearly see that the $p$-version shows a high rate of convergence and provides much more accurate results than the $h$-version within the same computation time (finest resolution). Note that in the case of the adaptive

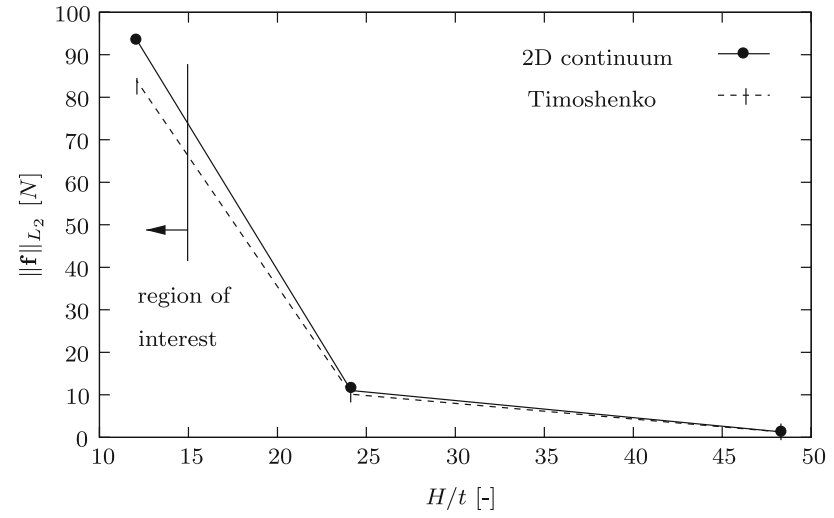

Fig. 9 Reaction forces depending on $H / t$; note that $H / t<15$ will be of interest within this work

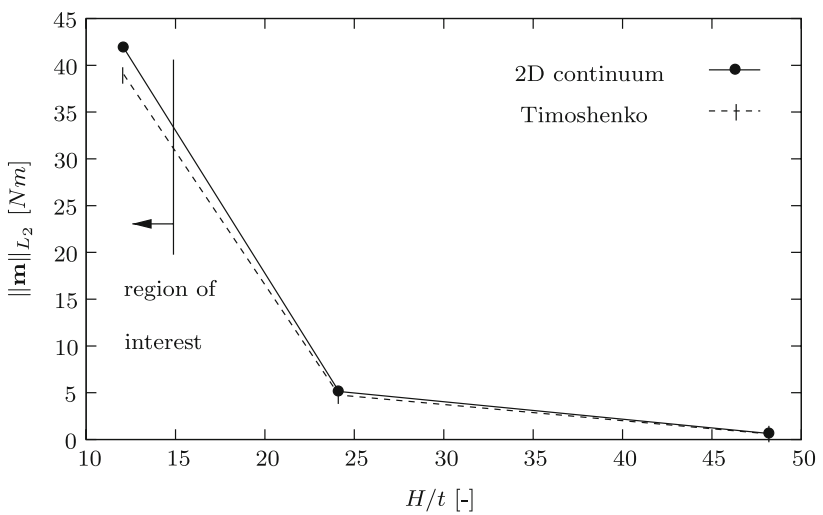

Fig. 10 Reaction moments depending on $H / t$; note that $H / t<15$ will be of interest within this work

$h$-refinement the time needed for mesh refinement is not included in the comparison.

Secondly, the influence of the wall-thickness is investigated: We reconsider the load case sketched in Fig. 7. Figures 9 and 10 compare at one Dirichlet boundary the reaction forces/moments of our continuum $p$-FEA with a finite element Timoshenko beam approximation whereby the aspect ratio $H / t$ is varied.

The differences between both approaches are pronounced for small $H / t$ and tend to zero for higher $H / t$. Considering the range of interest with $H / t<15$ demonstrates that the proposed $2 \mathrm{D}$ continuum approach excludes model errors introduced by the Timoshenko beam theory.

\section{Numerical examples}

In this section numerical examples for the $\mathrm{FE}^{2}$ approach are presented. In the first subsection we consider a TVE and investigate the accuracy of a corresponding discretization with $p$-FEM. To this end we carry out a hierarchical $p$-refinement in order to find a reliable discretization of the 


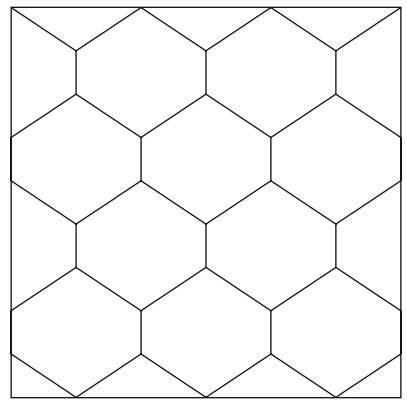

Fig. 11 Undisturbed TVE of size $\langle 1\rangle$

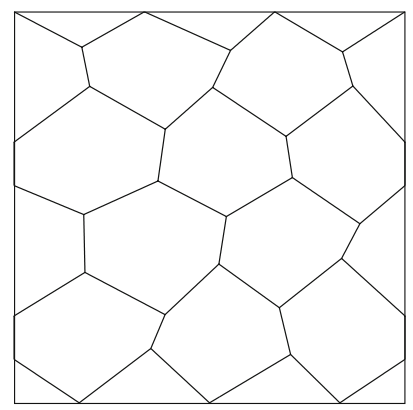

Fig. 12 TVE of size $\langle 1\rangle$ with random disturbances

TVE which will be applied in the subsequent subsections. The numerical examples include the application of the proposed $\mathrm{FE}^{2}$ framework to three specific examples: A uniaxial tension test, a simple shear test and a bending test.

\subsection{Testing volume element, discretization and boundary conditions}

The TVE corresponds to a section of a fully resolved foamlike structure and its size should be large enough to provide basic deformation mechanisms. In Fig. 11 an undisturbed TVE of size $\langle 1\rangle$ is depicted. $\mathrm{TVE}_{\langle 1\rangle}$ consists of hexagons whereby one hexagon is generated out of six beams. Note, that the hexagons have quadratic shapes, i.e. their beams do not have the same length. For a more realistic modeling of foam-like materials we introduce random disturbances to the geometry of the TVE. To this end, the original coordinates of the TVE are randomly disturbed, see Fig. 12.

These random disturbances can be regarded as imperfections which govern microstructural buckling patterns, i.e. the secondary path is entered directly and therefore a possibly occurring bifurcation problem is circumvented. A detailed description of microstructural buckling phenomena including bifurcation analysis is given e.g. in [24,33,35,44]. In order to study the influence of the TVE's size we introduce two additional TVEs of increasing size as shown in Figs. 13 and 14.

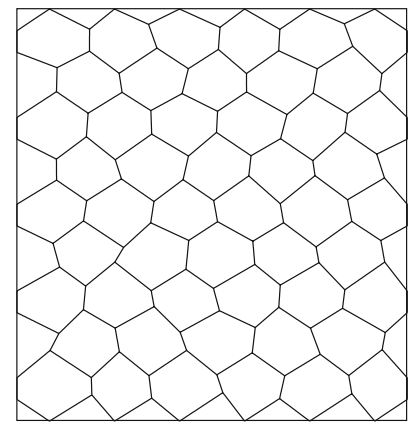

Fig. 13 TVE of size $\langle 2\rangle$ with random disturbances

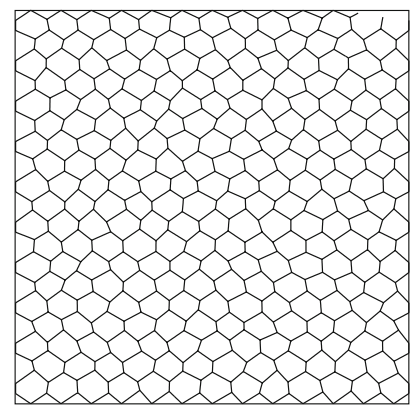

Fig. 14 TVE of size $\langle 4\rangle$ with random disturbances

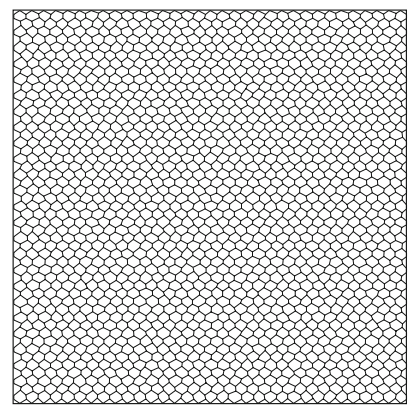

Fig. 15 Fully resolved foam-like structure with random disturbances

A fully resolved foam-like structure as shown in Fig. 15 will be used for creating reference solutions. Next, we consider the discretization of the TVE. In Fig. $16 \mathrm{TVE}_{<1>}$ being discretized with quadrilateral elements of high-order is depicted. By means of $\alpha_{m}$ the TVE's original $\mathbf{e}_{x_{m}} \mathbf{e}_{y_{m}}$-coordinate system can be rotated into the $\overline{\mathbf{e}}_{x_{m}} \overline{\mathbf{e}}_{y_{m}}$-coordinate system. The TVEs of size $\langle 2\rangle$ and $\langle 4\rangle$ are discretized analogously.

Since the $p$-version of the finite element method allows to use continuum elements with high aspect ratio, the TVE is meshed by two-dimensional beam-like elements (Fig. 16, B) that are connected by trapezoidal elements (Fig. 16, A). Two different types of boundary conditions are defined: A hard support uses the element's edge for setting the displacement and therefore corresponds to prescribing both translational and rotational degrees of freedom as in the classical beam 


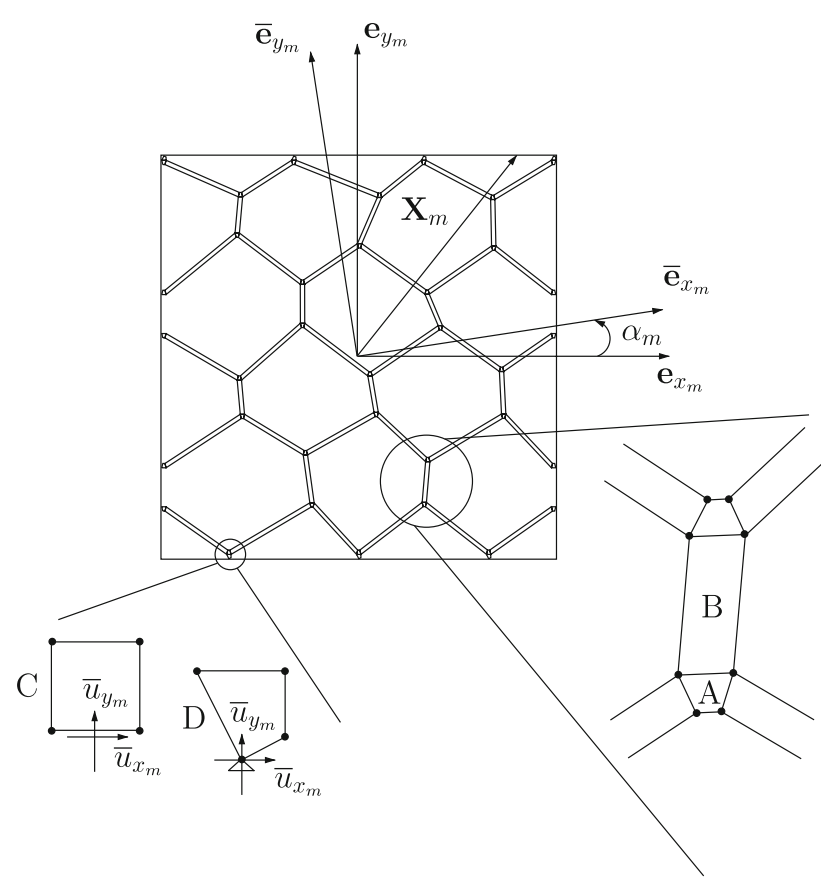

Fig. 16 Discretized $\mathrm{TVE}_{\langle 1\rangle}$ with random disturbances

theory (Fig. 16, C); a soft support uses only one single node and corresponds therefore to a pin support (Fig. 16, D). For the beam-like elements an anisotropic Ansatz in the local element directions (refer to Sect. 3.2) is chosen, for the other elements the Ansatz is isotropic, i.e. $p_{e}=q_{e}$.

Next, the accuracy for a $p$-refinement based on the tensor product space [38] is investigated. In [39] it has been demonstrated that a polynomial degree of $q_{e}=3$ in thickness direction is sufficient and yields a good accuracy. For the isotropic elements, we set $p_{e}=q_{e}=3$ in both local directions of the element. The question of how to choose the polynomial degree $p$ in the longitudinal direction of the beam-like elements is still open. To answer this question we apply an arbitrary state of deformation to $\mathrm{TVE}_{\langle 1\rangle}$ assuming soft support via (1), where

$\mathbf{F}_{M}=\left[\begin{array}{cc}0.1+1 & -0.13 \\ 0.16 & -0.15+1\end{array}\right]$

is fully populated and unsymmetric, leading to one nonlinear $\mathrm{BVP}_{m}$. The deformation is applied in 10 load steps, assuming a hyperelastic material behavior, see (7). We choose $\mu_{m}=$ 80.8 and $\Lambda_{m}=121.1 \mathrm{GPa}$, which corresponds to a compressible material. The polynomial degree $p=3, \ldots, 10$ is gradually increased. In Fig. 17 the strain energy $П$, obtained by integrating the strain energy density Eq. (7) in the reference configuration for all elements $n_{e}$

$\Pi=\sum_{e=1}^{n_{e}} \int_{\Omega_{e}} \Psi_{e} d \Omega_{e}$,

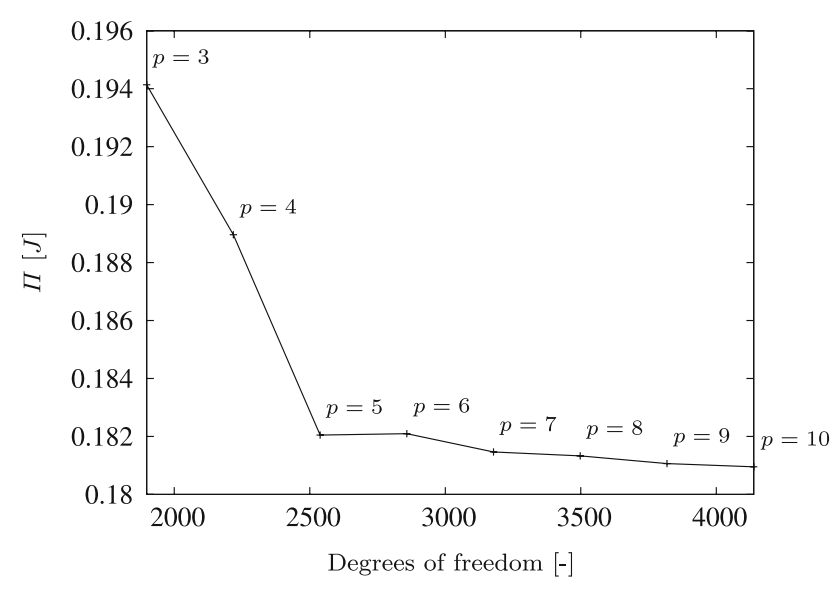

Fig. 17 Strain energy at the final load step plotted against the number of degrees of freedom

is plotted against the number of degrees freedom. From this it can be observed that $p=5$ yields a good accuracy. To be on the safe side we set $p=6$ for the subsequent numerical examples.

\subsection{Numerical experiments}

The numerical examples presented in this section are obtained by applying the proposed $\mathrm{FE}^{2}$ framework, as described in Sect. 2. On the macroscale we use bi-quadratic eight-noded Lagrangian finite elements each of with 4 Gaussian points. Note that the underintegration does not lead to any numerical problems. This is due to the fact that the zero energy modes, which might occur, are non-communicable; also refer to [26]. The load on the macroscale is applied incrementally.

At each Gaussian point of the macroscale a microstructure (TVE) is attached. For each macroscopic integration point the corresponding microstructure is randomly disturbed (as depicted in Fig. 12), yielding a statistical distribution of the disturbances which leads to small inhomogenities in macroscopic stresses and strains even when dealing with homogeneous macroscopic boundary conditions. Again, the material parameters in (7) are set to $\mu_{m}=80.8$ and $\Lambda_{m}=121.1 \mathrm{GPa}$.

\subsubsection{Uniaxial tension test}

We start with a macroscopic uniaxial tension test as shown in Fig. 18. To this end, the displacement $\bar{u}$ is split into a top and a bottom displacement, on the left boundary symmetry conditions are applied and the right boundary is free. The macroscopic mesh consists of $10 \times 10$ bi-quadratic finite elements. The width of the mesh is $B=2 \mathrm{~mm}$ and the height is $H=2 \mathrm{~mm}$.

A series of $\mathrm{FE}^{2}$ computations for a varying size of the TVE is performed and the results are compared to a reference solution. In the $\mathrm{FE}^{2}$ computations $\alpha_{m}$ is set to $0^{\circ}$ for all microstructures. The reference solution is obtained by using 

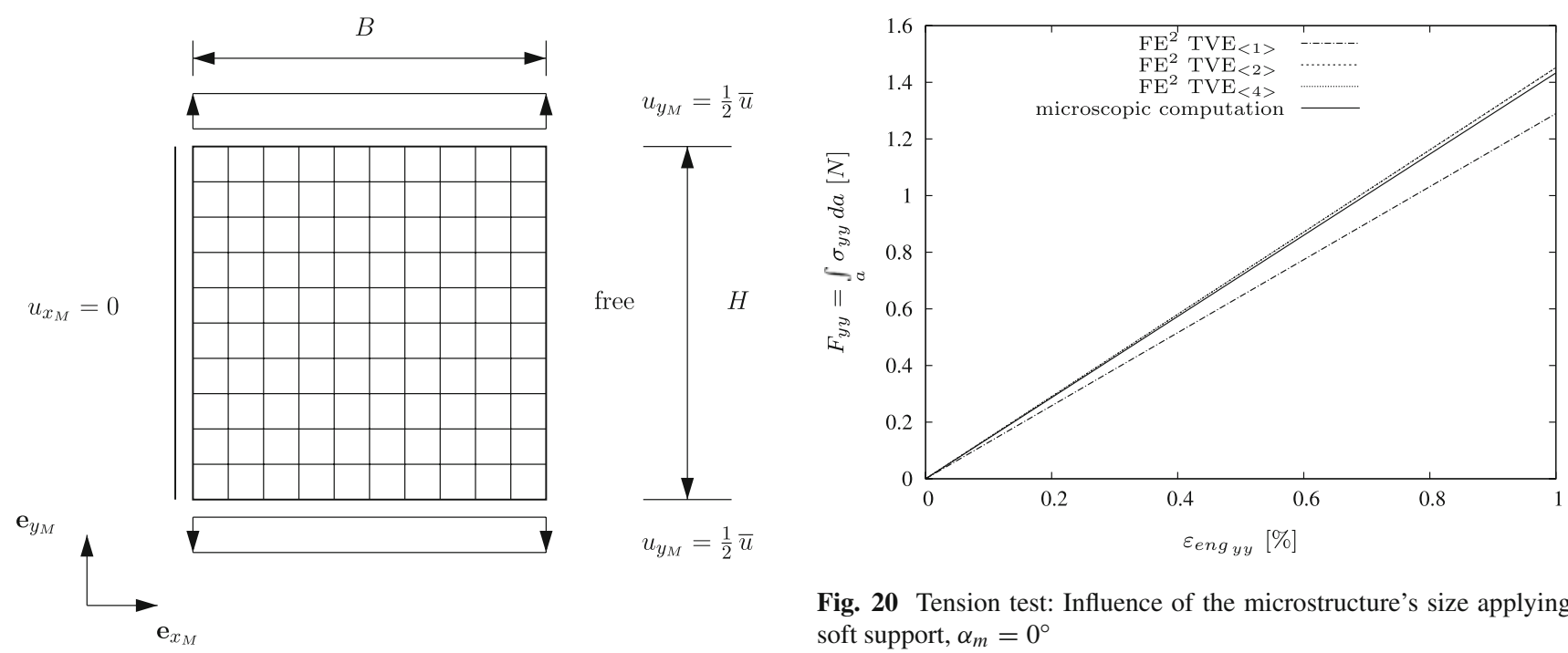

Fig. 18 Uniaxial tension test

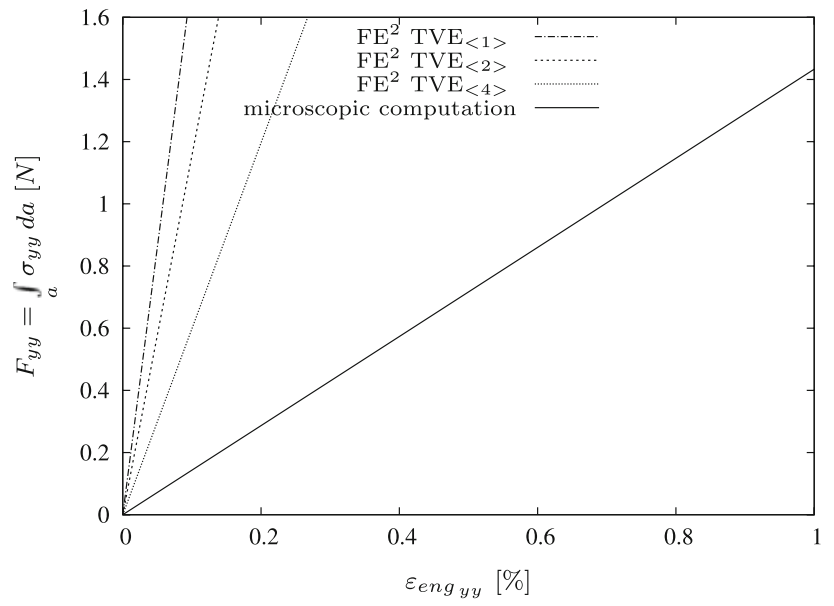

Fig. 19 Tension test: Influence of the microstructure's size applying hard support, $\alpha_{m}=0^{\circ}$

the fully resolved microstructure (microscopic computation) applying 2D continuum $p$-elements, refer to Fig. 15. Note, that nearly no difference between soft and hard support conditions for the reference solutions was observed for moderate strains $(\leq 10 \%)$. This fact can be explained by St. Venant's principle: The boundary layers are very small compared to the whole structure and therefore do not influence the overall mechanical behavior. As Dirichlet boundary conditions we chose hard support conditions.

In a first step, the influence of the size of the microstructure (TVE) is investigated in the small strain region for both types of boundary conditions.

In Fig. 19 the results for the hard support conditions are compared. To this end, the resulting force $F_{y y}=\int_{a} \sigma_{y y} d a$ is plotted against the strain $\varepsilon_{\mathrm{eng}}$. From this it can be observed that the $\mathrm{FE}^{2}$ computation with hard support yields a very stiff behavior even for $\mathrm{TVE}_{\langle 4\rangle}$. The reason for this stiffening effect

Fig. 20 Tension test: Influence of the microstructure's size applying soft support, $\alpha_{m}=0^{\circ}$

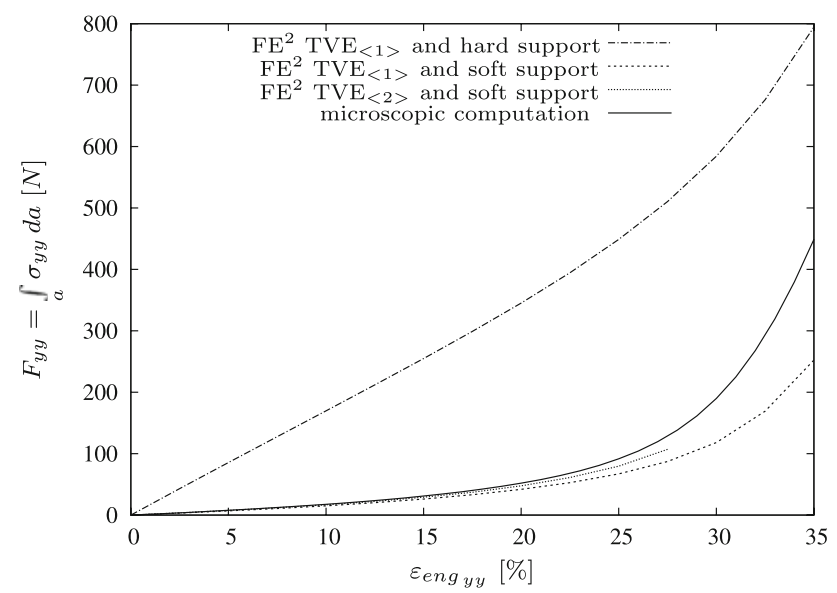

Fig. 21 Tension test: Load-engineering-strain curve for orientation $\alpha_{m}=0^{\circ}$

lies in the accumulation of strong boundary layer effects within each TVE. Note, that in this case a smaller TVE yields a stiffer mechanical behavior, see [3].

For soft support conditions a much better agreement with the reference solution for an increasing size of the microstructure can be observed, see Fig. 20. In fact, for $\mathrm{TVE}_{\langle 4\rangle}$ there is nearly no difference to the microscopic computation.

Now, we investigate the influence of large strains. In Fig. 21 the macroscopic load-engineering-strain curve ( $F_{y y}$ vs. $\varepsilon_{\text {eng }}$ ) is depicted.

From this it is evident that in the case of a hard support the stiffness is dramatically overestimated as expected $\left(\mathrm{FE}^{2}\right.$ $\mathrm{TVE}_{\langle 1\rangle}$ and hard support). Considering a soft support the $\mathrm{FE}^{2}$ computation agrees well with the curve of a reference solution until a strain of $\varepsilon_{\mathrm{eng}_{y y}} \approx 10 \%$ is reached. For higher strains the difference between both curves becomes more pronounced ( $\mathrm{FE}^{2}$ with $\mathrm{TVE}_{\langle 1\rangle}$ and soft support). If the size 


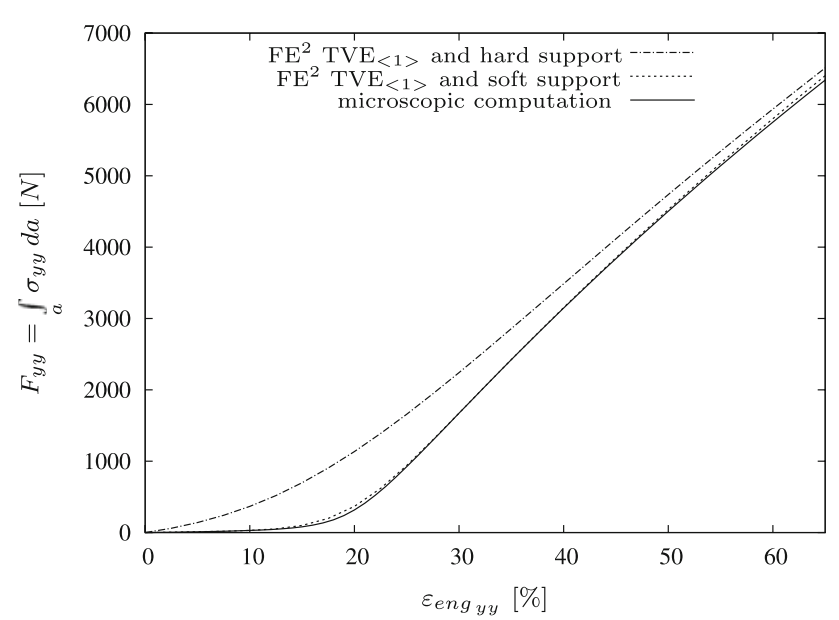

Fig. 22 Tension test: Load-engineering-strain curve for orientation $\alpha_{m}=90^{\circ}$

of the microstructure (TVE) is increased, a better agreement with the reference solution is obtained $\left(\mathrm{FE}^{2}\right.$ with $\mathrm{TVE}_{\langle 2\rangle}$ and soft support). This curve ends at a strain $\varepsilon_{\mathrm{eng}_{y y}} \approx 27.5 \%$ due to highly distorted elements.

To investigate the effect of anisotropy, the TVE is rotated by an angle of $\alpha_{m}=90^{\circ}$. Again, a reference solution is obtained based on a fully resolved structure, where the geometry and material parameters are chosen to be the same. The resulting curves are depicted in Fig. 22.

Again, it is observed that the $\mathrm{FE}^{2}$ computation with hard support differs significantly from the reference solution (microscopic approach), especially for small values of $\varepsilon_{\mathrm{eng}_{y y}}$. However, the soft support boundary conditions yield a very good agreement with the reference solution (microscopic computation). Therefore, we apply in the following only soft support conditions. These soft support conditions can be regarded as fluctuations relaxing the Dirichlet boundary conditions.

In Fig. 23 the transversal contraction $\frac{\Delta B}{\Delta H}$ is plotted against the engineering strain. In both cases a high transversal contraction is obtained, in the case of $\alpha_{m}=90^{\circ}$ one observes even a maximum. The result is not surprising, since in general foam-like structures exhibit high transversal contractions.

\subsubsection{Simple shear test}

In the simple shear test as depicted in Fig. 24 we apply periodic boundary conditions to the left and to the right boundary. One benefit of the periodic boundary conditions is, of course, the reduction in the number of elements, here only $1 \times 10$ elements are used. Note, that this shear test could also be performed with one single TVE. This would be more efficient but the small inhomogenities in macroscopic stresses and strains due to statistical disturbances varying between the TVEs would be excluded. The height of the shear layer is set to $H=2 \mathrm{~mm}$.

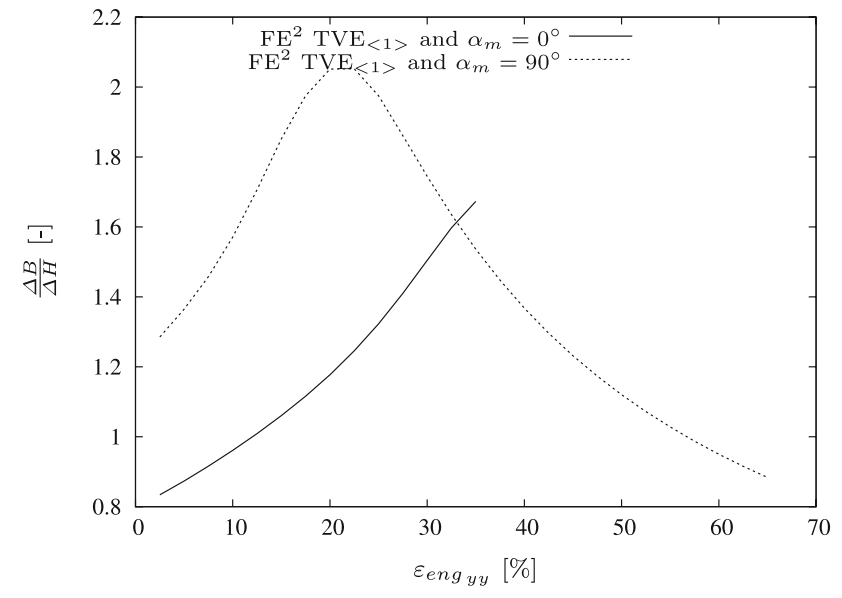

Fig. 23 Tension test: Transversal contraction for orientations $\alpha_{m}=0^{\circ}$ and $\alpha_{m}=90^{\circ}$

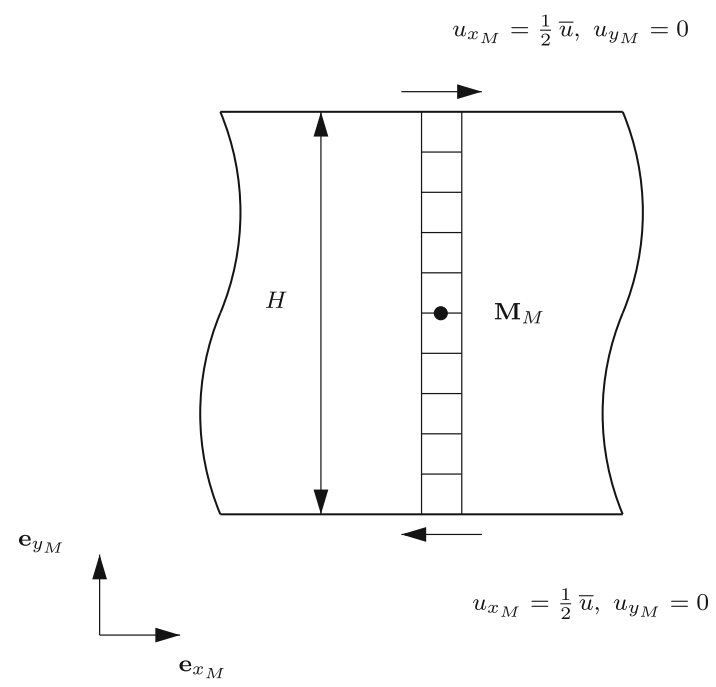

Fig. 24 Simple shear test with periodic boundary conditions

In Fig. 25 the macroscopic load-shearing-angle curve $\left(F_{x y}\right.$ vs. $\left.\tan (\gamma)=\frac{\bar{u}}{H}\right)$ and the deformed TVEs at the midpoint $\mathbf{M}_{M}$ (refer to Fig. 24) for two different orientations of the TVE are shown.

A nonlinear curve is observed until $\tan (\gamma) \approx 0.3$, which then transforms into a straight line. The pictures of the deformed TVEs indicate that there is a deformation-induced anisotropy, i.e. the gradual change of the load carrying behavior from bending to tension; compare with [24,42]. For a more quantitative investigation of this deformation-induced anisotropy, the principal angle for the Cauchy stress tensor $\sigma$ respectively the Euler-Almansi strain tensor $\mathbf{e}_{E A}=\left(\mathbf{I}-\left(\mathbf{F} \cdot \mathbf{F}^{T}\right)^{-1}\right)$

$\varphi_{\boldsymbol{\sigma}}=\frac{1}{2} \operatorname{atan}\left(\frac{2 \sigma_{x y}}{\sigma_{x x}-\sigma_{y y}}\right)$

$\varphi \mathbf{e}_{E A}=\frac{1}{2} \operatorname{atan}\left(\frac{2 \mathbf{e}_{E A x y}}{\mathbf{e}_{E A x x}-\mathbf{e}_{E A y y}}\right)$ 


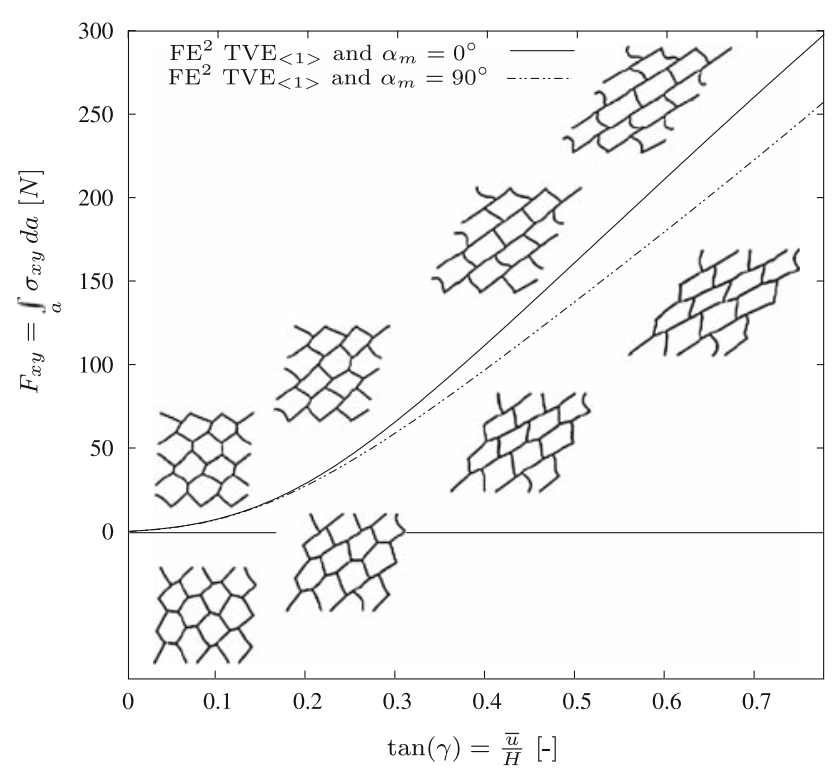

Fig. 25 Shear test: Load-shearing-angle curve for orientations $\alpha_{m}=$ $0^{\circ}$ and $\alpha_{m}=90^{\circ}$; for both orientations the deformed TVEs at the midpoint $\mathbf{M}_{M}$ (refer to Fig. 24) are plotted

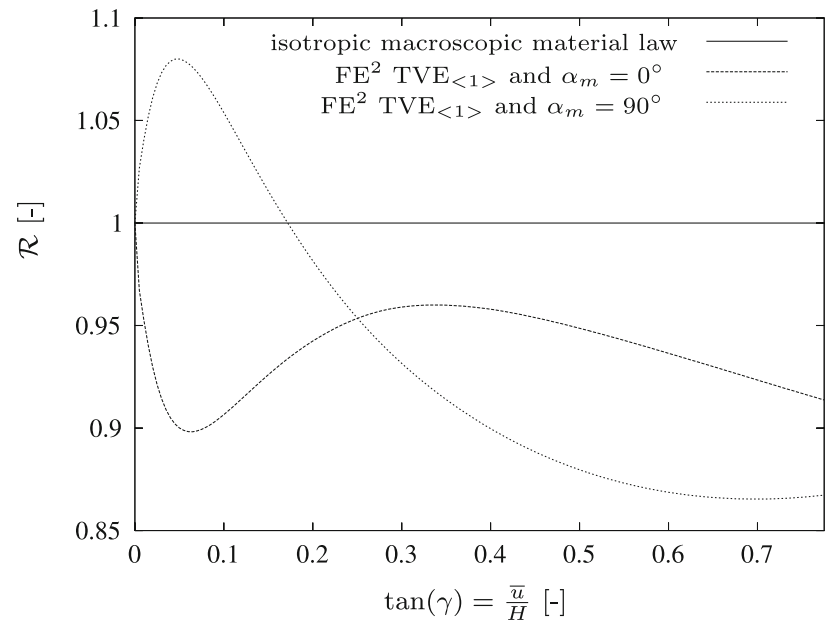

Fig. 26 Shear test: Ratio-shearing-angle curve for orientations $\alpha_{m}=0^{\circ}$ and $\alpha_{m}=90^{\circ}$

are introduced. We define the ratio between $\varphi_{\boldsymbol{\sigma}}$ and $\varphi_{\mathbf{e}_{E A}}$ as

$\mathcal{R}=\frac{\varphi_{\boldsymbol{\sigma}}}{\varphi_{\mathbf{e}_{E A}}}$.

Note, that for an isotropic material law $\mathcal{R}=$ constant $=1$. In Fig. 26 the ratio-shearing-angle curves $\left(\mathcal{R}\right.$ vs. $\left.\tan (\gamma)=\frac{\bar{u}}{H}\right)$ are plotted for the midpoint $\mathbf{M}_{M}$ of the structure. A curve obtained by using the isotropic macroscopic material law as stated in (7) is included, too.

In contrast to the computation based on this isotropic macroscopic material law, the ratios of the $\mathrm{FE}^{2}$ computations are not constant which is an indicator for an anisotropic material behavior.

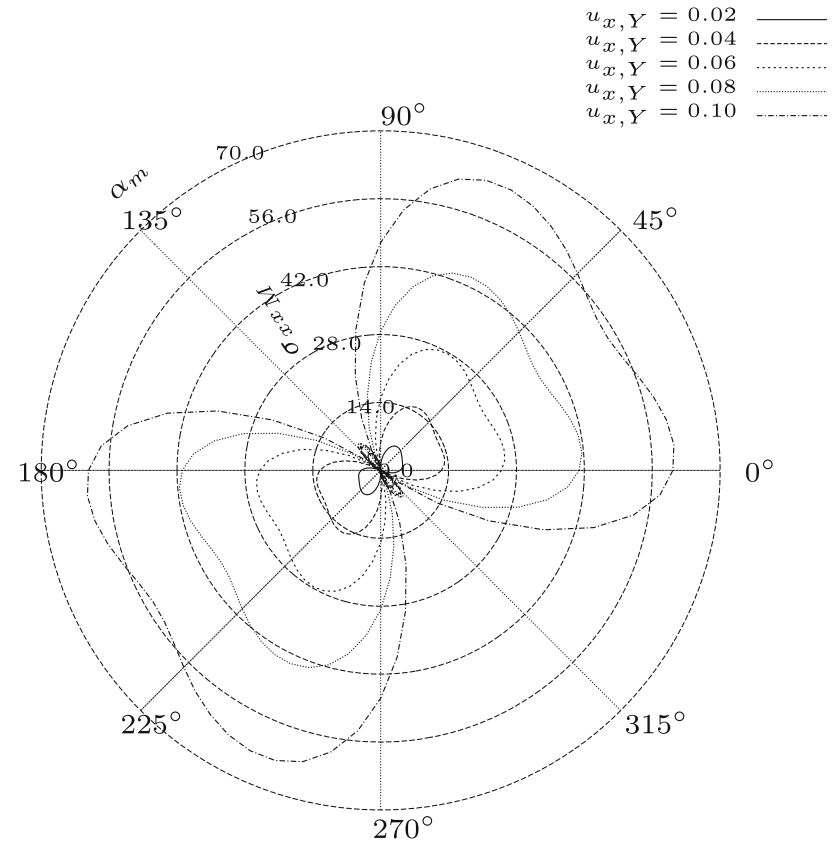

Fig. 27 Stresses $\sigma_{x x_{M}}$ for $\mathrm{TVE}_{\langle 1\rangle}$ orientations $\alpha_{m}=0^{\circ}, \ldots, 360^{\circ}$ and $u_{x, Y}=0.02, \ldots, 0.1$

Next we consider the influence of the orientation $\alpha_{m}$ of the TVEs with respect to the resulting stress, compare with [42]. We apply for each $\alpha_{m}$ the same state of deformation (i.e. shearing) to $\mathrm{TVE}_{\langle 1\rangle}, \mathrm{TVE}_{\langle 2\rangle}$, and $\mathrm{TVE}_{\langle 4\rangle}$

$\mathbf{F}_{M}=\left[\begin{array}{cc}1+0.0 & 0.1 \\ 0.0 & 1+0.0\end{array}\right]$

which is applied in five load steps. In Figs. 27 to 35 the components of the homogenized Cauchy stresses are plotted using the same scale for all TVEs. Obviously, they obey an anisotropic behavior, i.e. they depend on $\alpha_{m}$, and their shape depends on the TVE size. Note, that there is no significant influence of the disturbances on the anisotropy, i.e. the disturbances are too small to regularize the mechanical behavior. Comparing the maximum stress values for the three TVEs one realizes that they decrease with increasing TVE size. This decrease is directly associated with the application of displacement boundary conditions: If the TVE size is increased the stresses will converge to a limit, i.e. the TVE is representative for the material under consideration and can therefore be termed RVE $[23,27]$. Note however that our TVEs are too small to be representative, since no convergence in stresses is obtained, yet.

Another interesting point is that although we are dealing with a shear test the normal stresses $\sigma_{x x_{M}}$ and $\sigma_{y y_{M}}$ are dominant. This observation is directly connected to the inherent properties of the microstructure (TVE). 


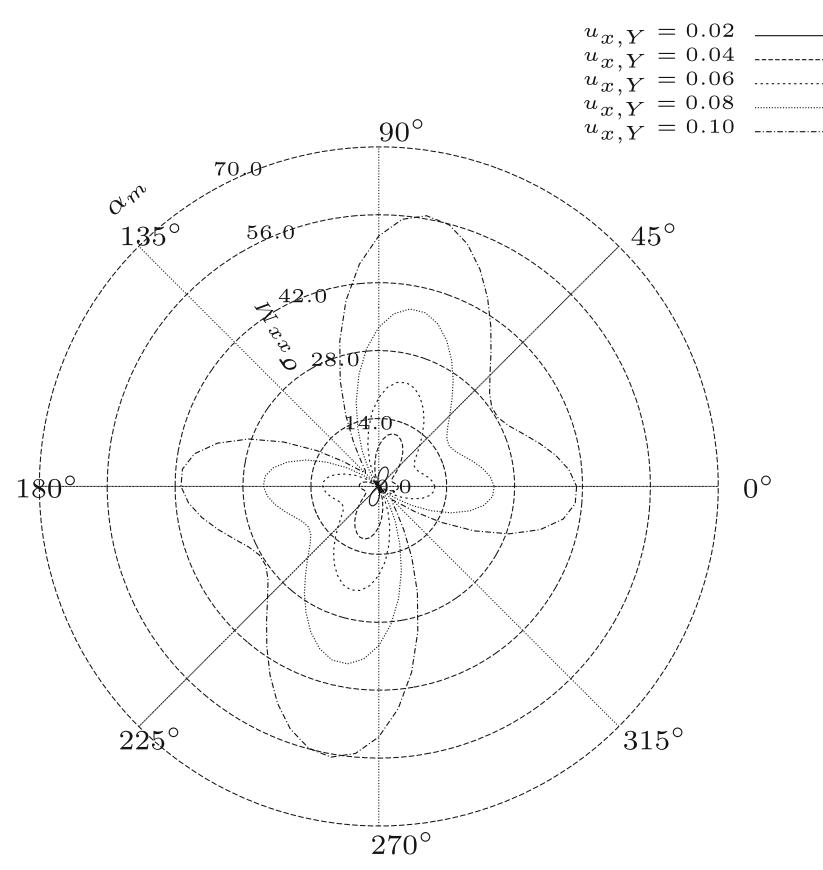

Fig. 28 Stresses $\sigma_{x x_{M}}$ for $\operatorname{TVE}_{\langle 2\rangle}$ orientations $\alpha_{m}=0^{\circ}, \ldots, 360^{\circ}$ and $u_{x, Y}=0.02, \ldots, 0.1$

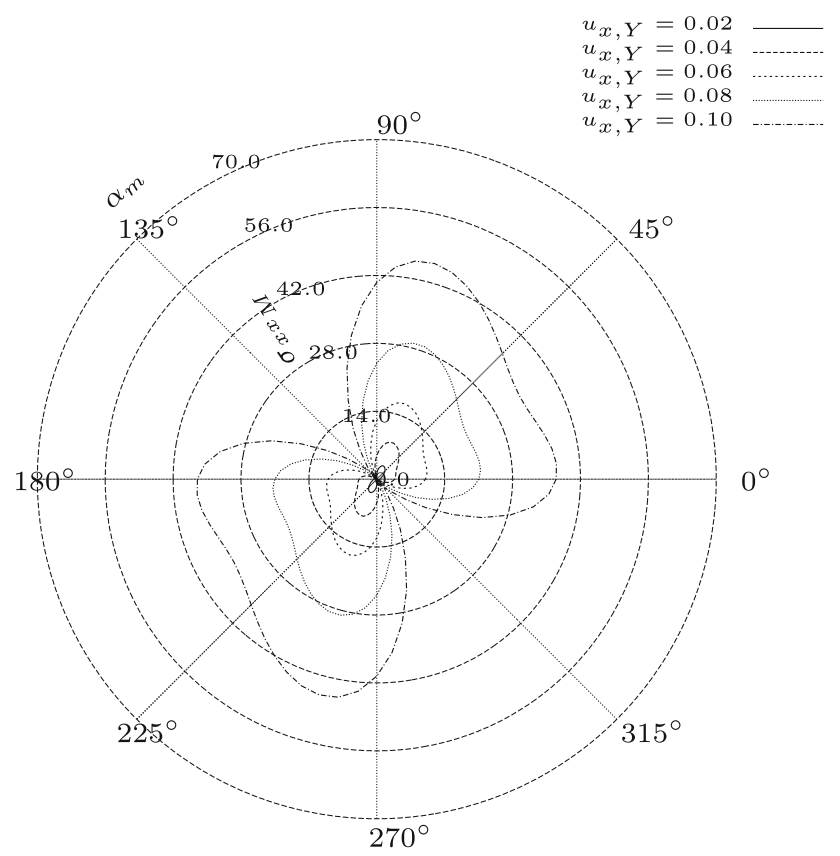

Fig. 29 Stresses $\sigma_{x x_{M}}$ for $\mathrm{TVE}_{\langle 4\rangle}$ orientations $\alpha_{m}=0^{\circ}, \ldots, 360^{\circ}$ and $u_{x, Y}=0.02, \ldots, 0.1$

In Figs. 36, 37, and 38 the principal angle computed from the Cauchy stress tensor is plotted. Again, an anisotropic behavior depending on $\alpha_{m}$ can be observed.

\subsubsection{Bending test}

In the macroscopic bending test a beam-like structure is symmetrically loaded by a prescribed displacement. Due to

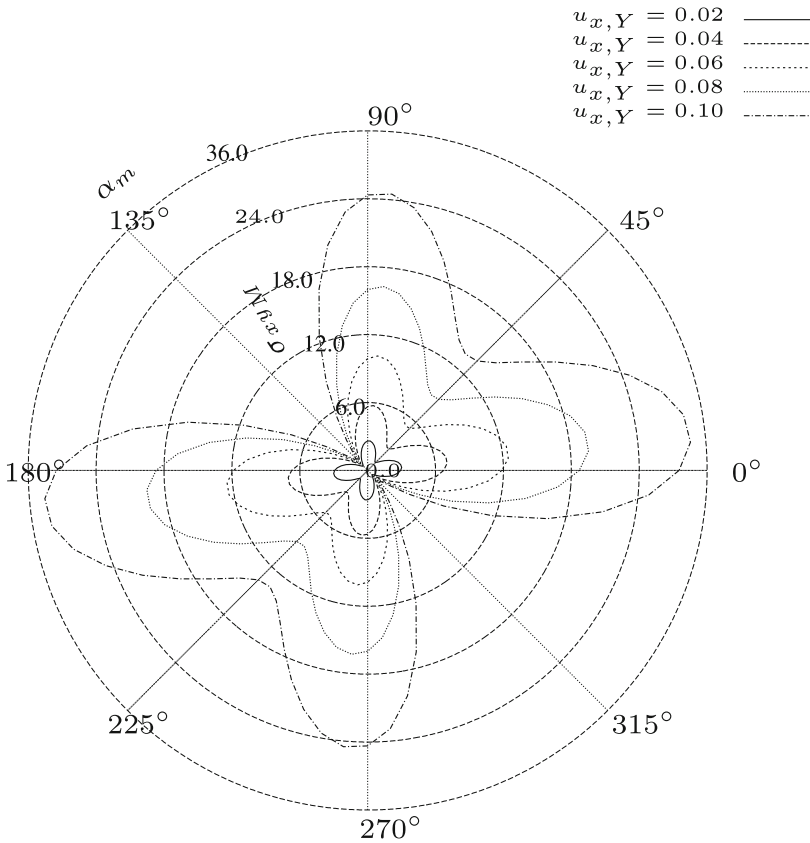

Fig. 30 Stresses $\sigma_{x y_{M}}$ for $\mathrm{TVE}_{\langle 1\rangle}$ orientations $\alpha_{m}=0^{\circ}, \ldots, 360^{\circ}$ and $u_{x, Y}=0.02, \ldots, 0.1$

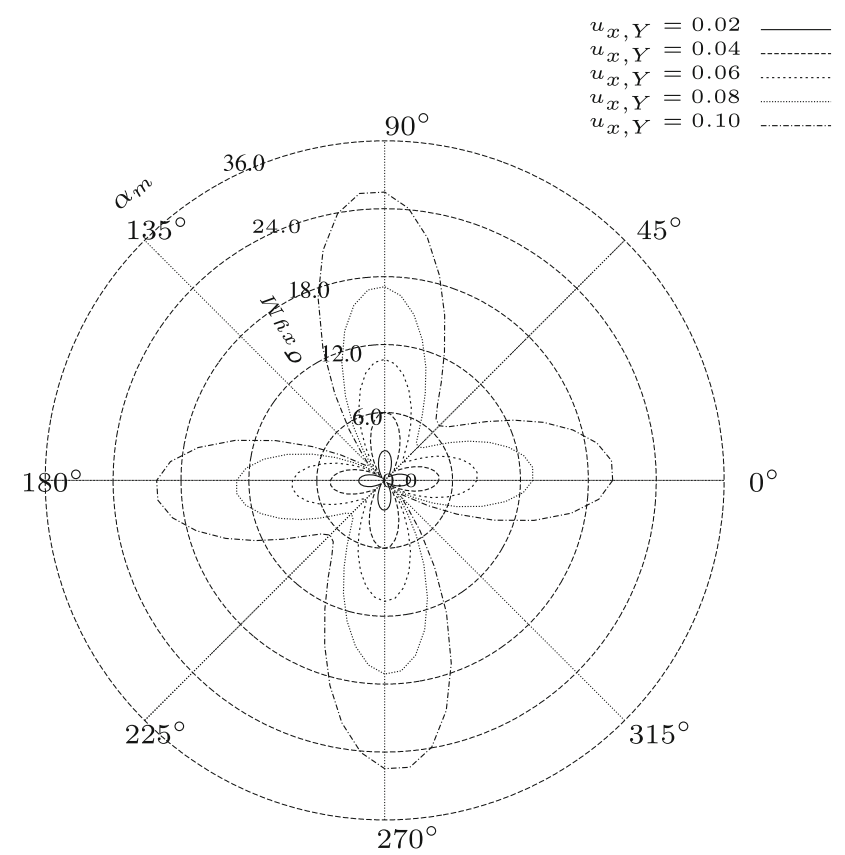

Fig. 31 Stresses $\sigma_{x y_{M}}$ for $\operatorname{TVE}_{\langle 2\rangle}$ orientations $\alpha_{m}=0^{\circ}, \ldots, 360^{\circ}$ and $u_{x, Y}=0.02, \ldots, 0.1$

symmetry only half of the system has to be modeled, see Fig. 39. The macroscopic mesh consists of $20 \times 5$ elements. The length is $L=8 \mathrm{~mm}$ and the height is $H=2 \mathrm{~mm}$. Therefore, the aspect ratio $L / H$ of the beam equals 4 . This example is referred to as inhomogeneous since bending is in general a combination of shear, tension and compression. 


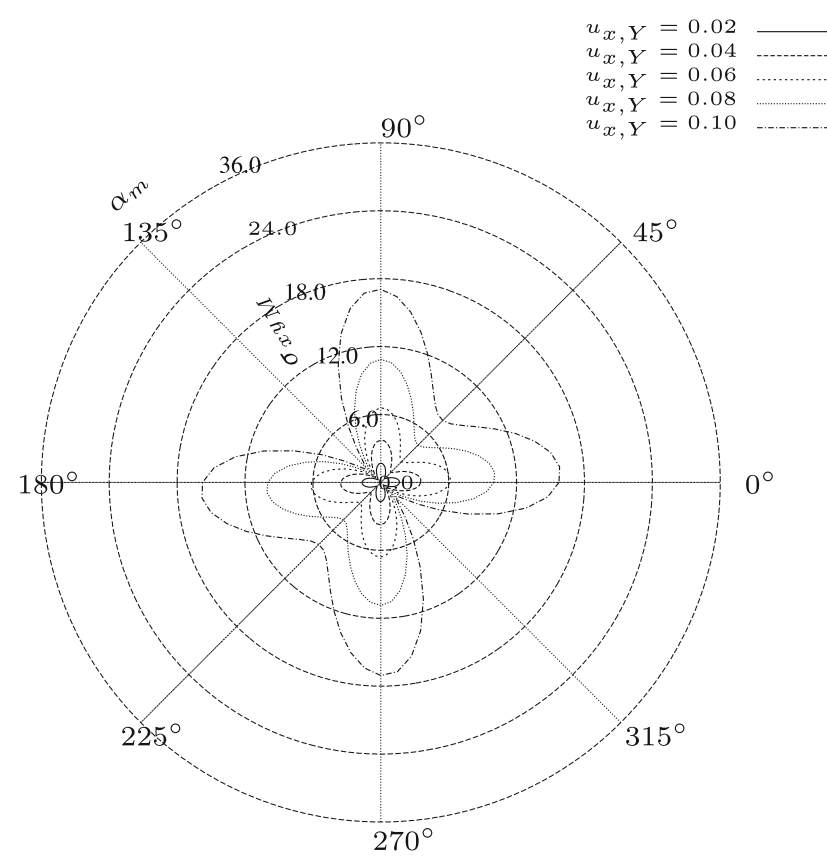

Fig. 32 Stresses $\sigma_{x y_{M}}$ for $\operatorname{TVE}_{\langle 4\rangle}$ orientations $\alpha_{m}=0^{\circ}, \ldots, 360^{\circ}$ and $u_{x, Y}=0.02, \ldots, 0.1$

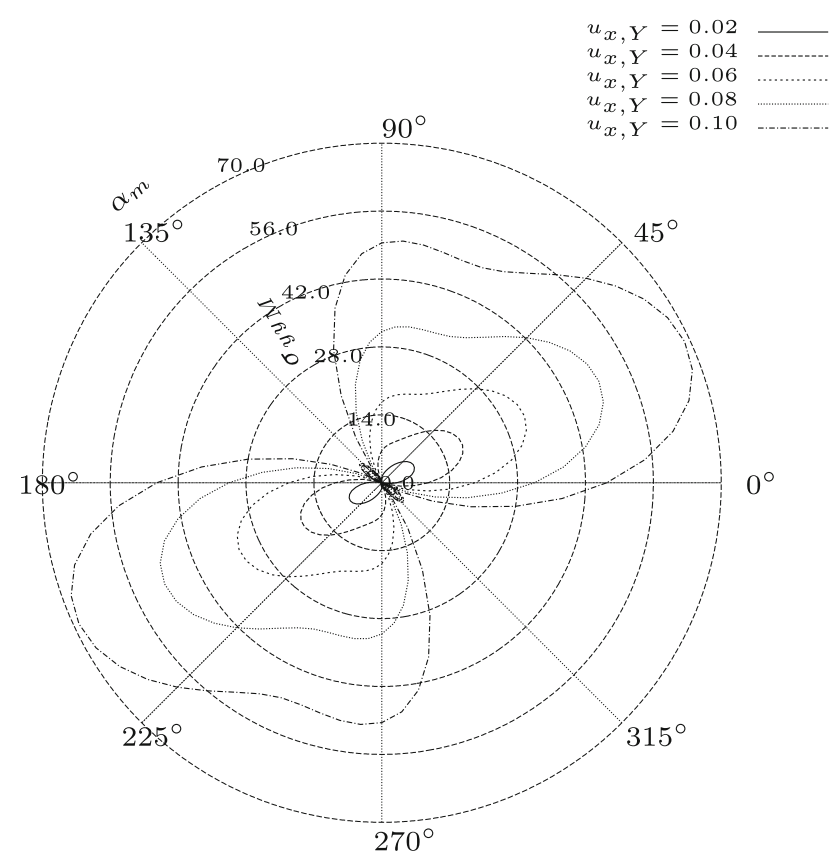

Fig. 33 Stresses $\sigma_{y y_{M}}$ for $\mathrm{TVE}_{\langle 1\rangle}$ orientations $\alpha_{m}=0^{\circ}, \ldots, 360^{\circ}$ and $u_{x, Y}=0.02, \ldots, 0.1$

The macroscopic load-displacement curves $\left(F_{x y}\right.$ vs. $\left.\bar{u}\right)$ for two different TVE orientations $\alpha_{m}=0^{\circ}$ and $\alpha_{m}=90^{\circ}$ are shown in Fig. 40.

From this it is evident that the load-displacement curves obey a nonlinear behavior. Let us focus on the displacement $u_{y_{M}}$ at the midline of the macrostructure which is evaluated at the cutline indicated in Fig. 39. Figure 41 shows $u_{y_{M}}$ for

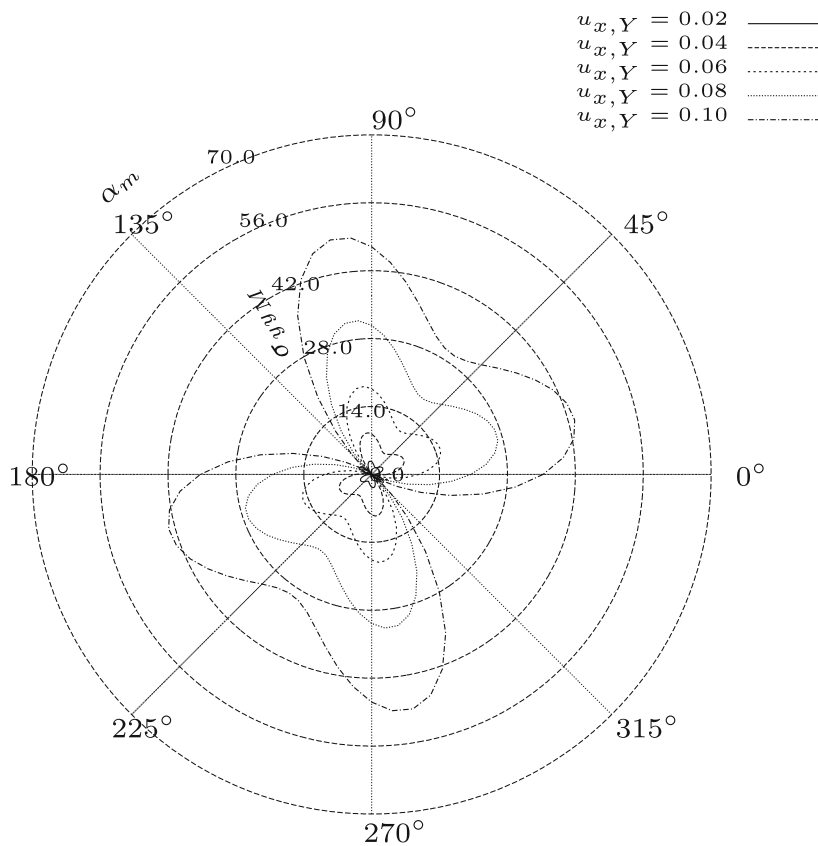

Fig. 34 Stresses $\sigma_{y y_{M}}$ for $\operatorname{TVE}_{\langle 2\rangle}$ orientations $\alpha_{m}=0^{\circ}, \ldots, 360^{\circ}$ and $u_{x, Y}=0.02, \ldots, 0.1$

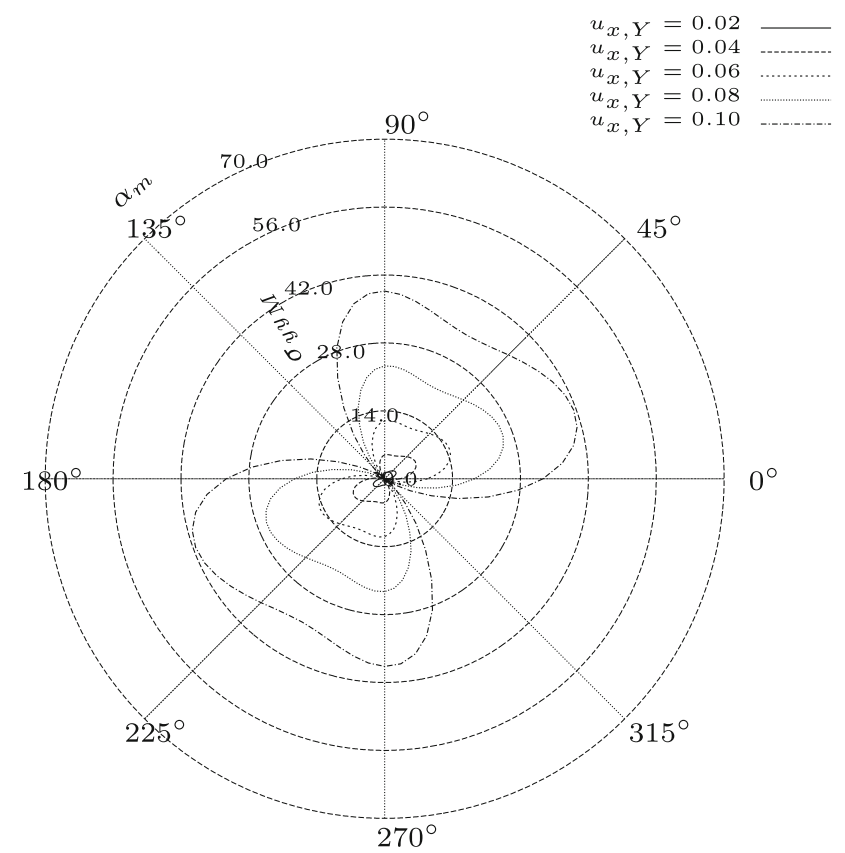

Fig. 35 Stresses $\sigma_{y y_{M}}$ for $\mathrm{TVE}_{\langle 4\rangle}$ orientations $\alpha_{m}=0^{\circ}, \ldots, 360^{\circ}$ and $u_{x, Y}=0.02, \ldots, 0.1$

$\bar{u}=1.0$ over the beam's length for the two different orientations of the TVE and additionally for a computation based on an isotropic macroscopic material law.

Whereas the rotation of the TVE by $90^{\circ}$ is almost without effect, a very small difference between the results of the $\mathrm{FE}^{2}$ approach and the isotropic macroscopic material law can be observed. To give an impression about the computational 


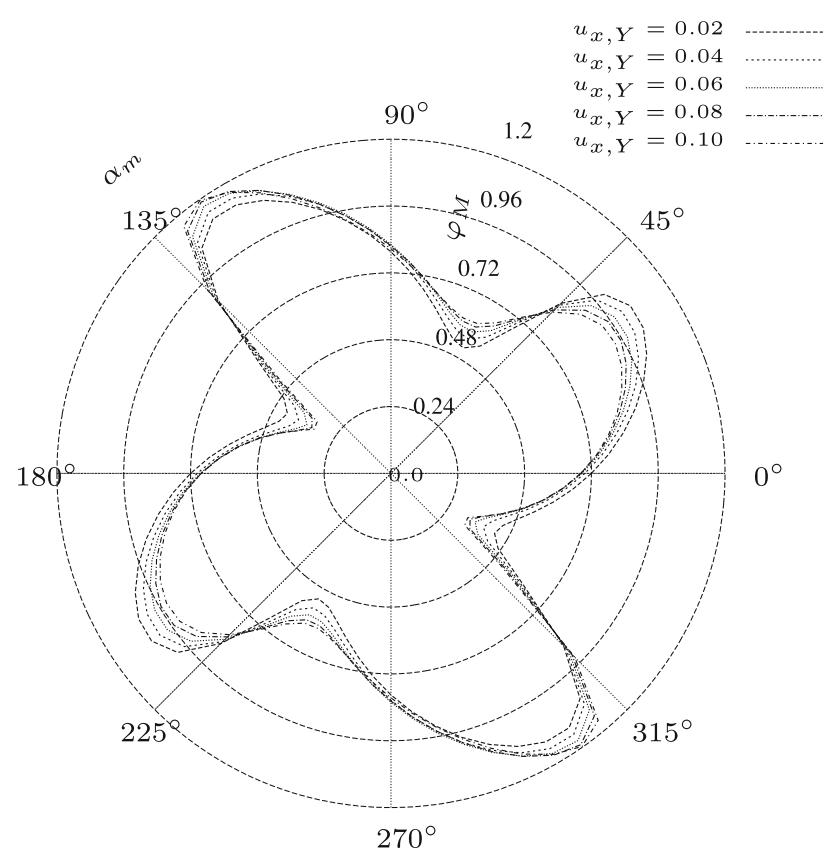

Fig. 36 Principal angle $\varphi_{M}$ for $\operatorname{TVE}_{\langle 1\rangle}$ orientations $\alpha_{m}=0^{\circ}, \ldots$, $360^{\circ}$ and $u_{x, Y}=0.02, \ldots, 0.1$

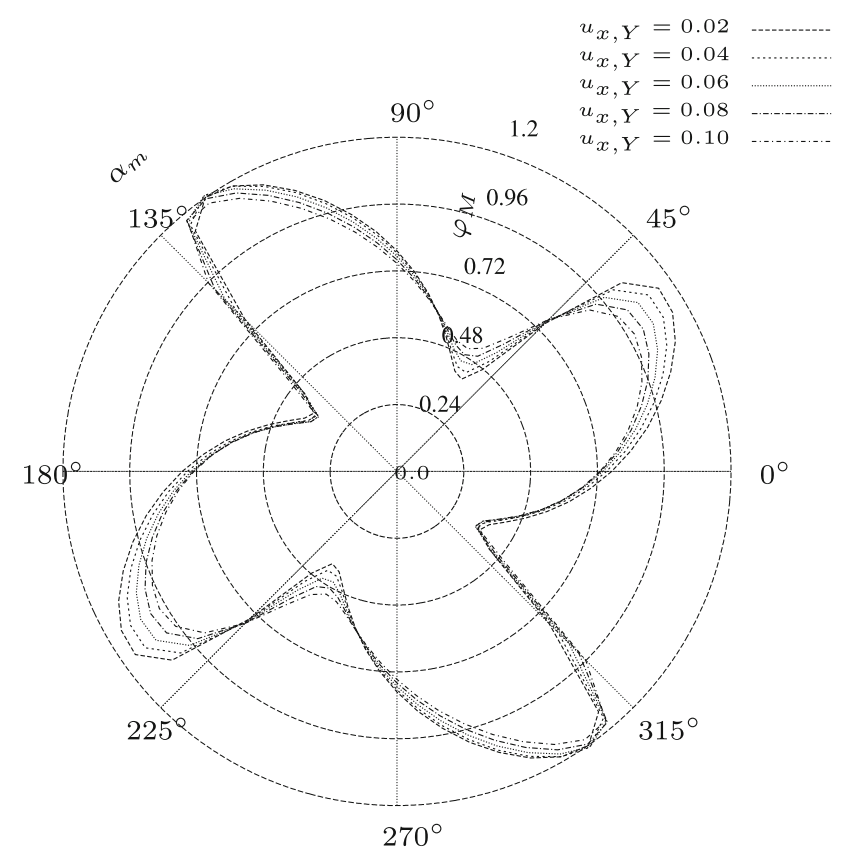

Fig. 37 Principal angle $\varphi_{M}$ for $\operatorname{TVE}_{\langle 2\rangle}$ orientations $\alpha_{m}=0^{\circ}, \ldots$, $360^{\circ}$ and $u_{x, Y}=0.02, \ldots, 0.1$

effort: One bending test computation took around two days on the above mentioned cluster.

\section{Conclusions and outlook}

In this contribution we presented a two-dimensional firstorder $\mathrm{FE}^{2}$ framework suited for geometrically nonlinear

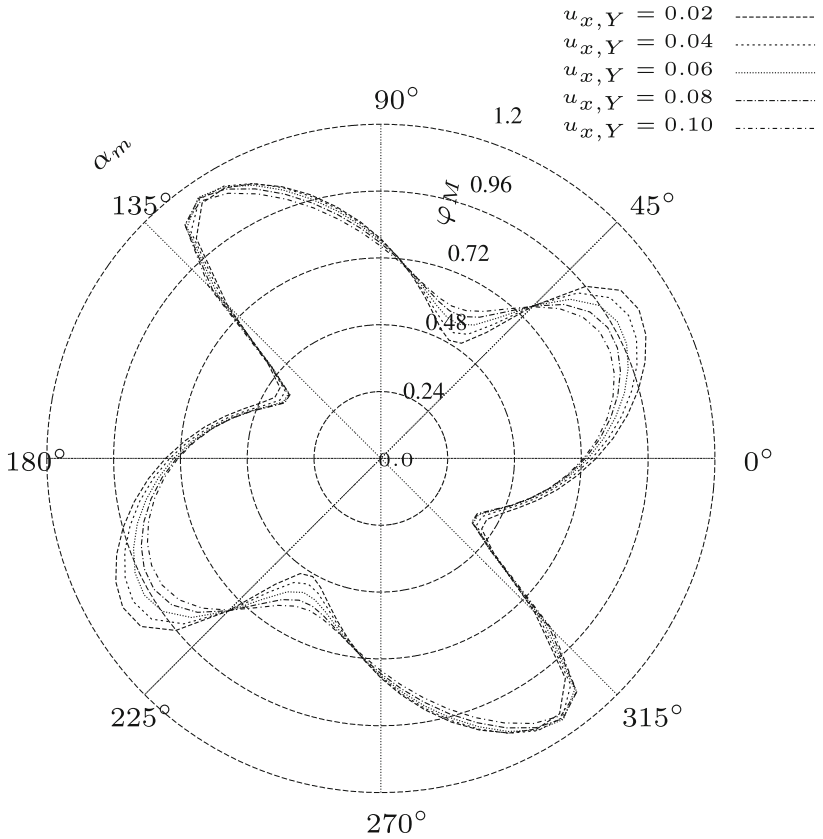

Fig. 38 Principal angle $\varphi_{M}$ for $\operatorname{TVE}_{\langle 4\rangle}$ orientations $\alpha_{m}=0^{\circ}, \ldots$, $360^{\circ}$ and $u_{x, Y}=0.02, \ldots, 0.1$

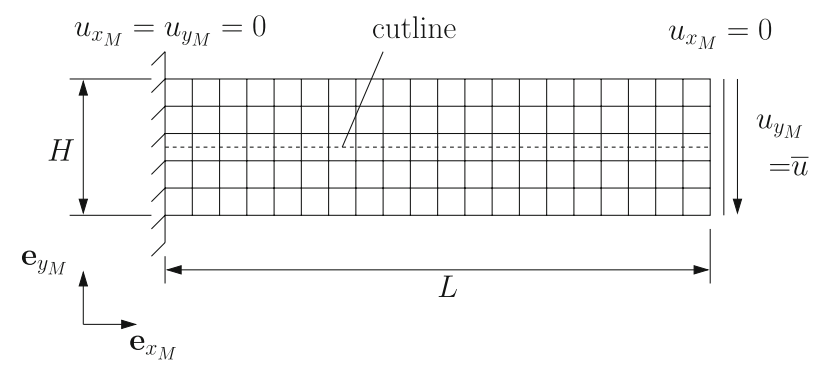

Fig. 39 Bending test

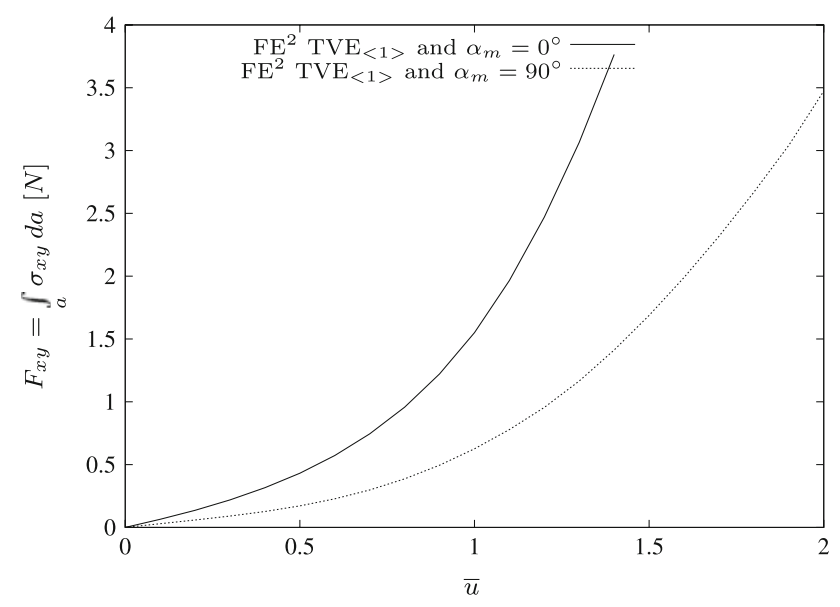

Fig. 40 Bending test: Load-displacement curve for TVE orientations $\alpha_{m}=0^{\circ}$ and $\alpha_{m}=90^{\circ}$

computations of foam-like structures. Since the $\mathrm{FE}^{2}$ approach is parallel by its nature, the presented framework makes use of distributed computing, which reduces the overall 


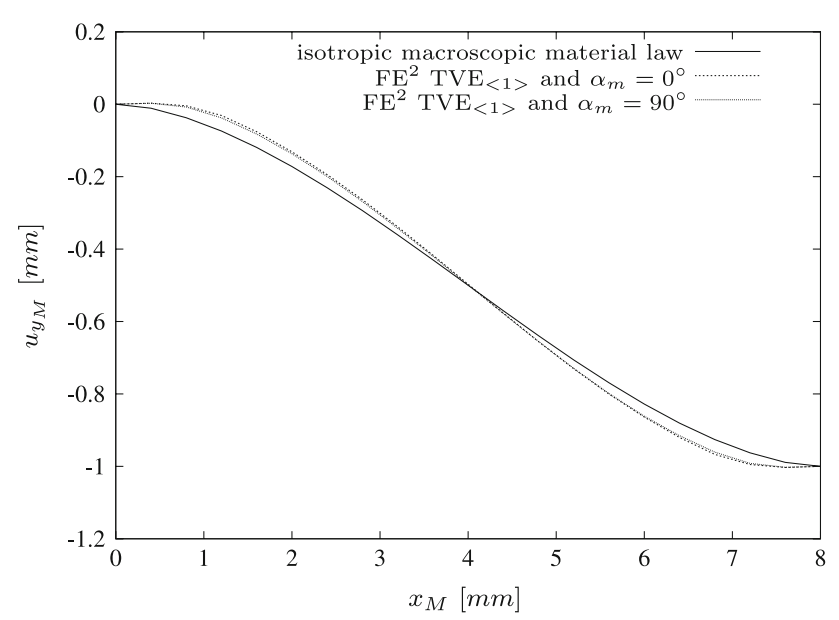

Fig. 41 Bending test: Midline displacement $u_{y_{M}}$ for $\bar{u}=1.0$

computation time. On the microscale two-dimensional continuum high-order finite elements, which are known to be highly efficient, are applied to discretize the microstructure's cell walls. In our numerical examples we demonstrated, that the presented framework is able to capture the macroscopic load-displacement behavior and the deformation-induced anisotropy (i.e. changing the load carrying behavior from bending to tension) within the microstructures. For the tension test we compared the results obtained by $\mathrm{FE}^{2}$ computations with a microscopic approach for two types of boundary conditions: Soft support and hard support. A good agreement is achieved when applying soft support conditions. In the macroscopic shear test we investigated in detail the deformation-induced anisotropy and demonstrated the general anisotropy of our microstructure by considering single TVEs. The bending test was chosen to show that also inhomogeneous macroscopic computations can be performed with the proposed method.

The application of periodic boundary conditions on the microscale would help to further increase the quality of the results. By replacing the discrete Newton-Raphson scheme on the macrolevel with one utilizing a consistent tangent, the overall computational effort could be reduced further. In this case the microstructure's consistent material tangents would have to be computed as proposed by Schröder [36]. In order to validate our results we have to enhance our scheme to three dimensions [19]. Using extended continua (both linear and nonlinear) on the macroscale and modifying the projection and homogenization procedure will enable us to include and investigate also size effects.

Acknowledgments The support by the Deutsche Forschungsgemeinschaft (DFG) is gratefully acknowledged (RA 624/16-1, DI 430/7-1). The authors wish to thank the reviewers for their helpful comments.
Open Access This article is distributed under the terms of the Creative Commons Attribution Noncommercial License which permits any noncommercial use, distribution, and reproduction in any medium, provided the original author(s) and source are credited.

\section{References}

1. Anderson WB, Lakes RS (1994) Size effects due to Cosserat elasticity and surface damage in closed-cell polymethacrylimide foam. J Mat Sci 29:6413-6419

2. Bonet J, Wood RD (1997) Nonlinear continuum mechanics for finite element analysis. Cambridge University Press, New York

3. Diebels S, Steeb H (2002) The size effect in foams and its theoretical and numerical investigation. Proc R Soc Lond A 458: 2869-2883

4. Diebels S, Steeb H (2003) Stress and couple stress in foams. Comp Mat Sci 28:714-722

5. Düster A (2001) High order finite elements for three-dimensional, thin-walled nonlinear continua. PhD thesis, Lehrstuhl für Bauinformatik, Fakultät für Bauingenieur- und Vermessungswesen, Technische Universität München

6. Düster A, Bröker H, Rank E (2001) The p-version of the finite element method for three-dimensional curved thin walled structures. Int J Numer Meth Eng 52:673-703

7. Düster A, Hartmann S, Rank E (2003) p-fem applied to finite isotropic hyperelastic bodies. Comput Methods Appl Mech Eng 192:5147-5166

8. Düster A, Rank E (2001) The p-version of the finite element method compared to an adaptive h-version for the deformation theory of plasticity. Comput Methods Appl Mech Eng 190: 1925-1935

9. Düster A, Rank E (2002) A p-version finite element approach for two- and three-dimensional problems of the $J_{2}$ flow theory with non-linear isotropic hardening. Int J Numer Methods Eng 53:49-63

10. Düster A, Scholz D, Rank E (2007) $p q$-Adaptive solid finite elements for three-dimensional plates and shells. Comput Methods Appl Mech Eng 197:243-254

11. Ebinger T, Diebels S, Steeb H (2007) Numerical homogenization techniques applied to growth and remodelling phenomena. Comp Mech 39:815-830

12. Ebinger T, Steeb H, Diebels S (2004) Modeling macroscopic extended continua with the aid of numerical homogenization schemes. Comput Mat Sci 32:337-347

13. Eringen AC, Suhubi ES (1964) Nonlinear theory of simple microelastic solids-I. Int J Eng ng Sci 2:189-203

14. Eringen C (1999) Microcontinuum field theories, vol 1: foundations and solids. Springer, Berlin

15. Feyel F (1998) Application du calcul parallèle aux modèles à grand nombre de variables internes. PhD-thesis, Ecole National Supérieure des Mines de Paris, Paris, France

16. Feyel F (2003) A multilevel finite element method ( $\mathrm{FE}^{2}$ to describe the response of highly non-linear structures using generalized continua). Comput Methods Appl Mech Eng 192:3233-3244

17. Feyel F, Chaboche JL (2000) $\mathrm{FE}^{2}$ multiscale approach for modelling the elastoviscoplastic behaviour of long fiber SiC/Ti composite materials. Comput Methods Appl Mech Eng 183:309-330

18. Gibson LJ, Ashby MF (1997) Cellular solids: structure and properties. Cambridge solid state science series. Cambridge University Press, Cambridge

19. Hain M (2007) Computational homogenization of micro-structural Damage due to Frost in Hardened Cement Paste. PhD thesis, Institut für Baumechanik und Numerische Mechanik, Leibniz-Universität Hannover 
20. Hartmann S (2003) Finite-Elemente Berechnung inelastischer Kontinua-Interpretation als Algebro-Differentialgleichungssysteme. Postdoctoral thesis, Institut für Mechanik, Universität Kassel

21. Heißerer U, Hartmann S, Düster A, Bier W, Yosibash Z, Rank E (2008) $p$-fem for finite deformation powder compaction. Comput Methods Appl Mech Eng 197:727-740

22. Heißerer U, Hartmann S, Düster A, Yosibash Z (2008) On volumetric locking-free behavior of p-version finite elements under finite deformations. Commun Numer Methods Eng 24:1019-1032

23. Hill R (1963) Elastic properties of reinforced solids: some theoretical principles. J Mech Phys Solids 11:357-372

24. Hohe J, Becker W (2003) Effective mechanical behavior of hyperelastic honeycombs and two-dimensional model foams at finite strain. Int J Mech Sci 45:891-913

25. Kafadar CB, Eringen C (1971) Micropolar media-I: the classical theory. Int J Eng Sci 9:271-305

26. Koschnick F (2004) Geometrische Locking-Effekte bei Finiten Elementen und ein allgemeines Konzept zu ihrer Vermeidung. PhD thesis, Lehrstuhl für Statik, Technische Universität München

27. Kouznetsova VG (2002) Computational homogenization for the multi-scale analysis of multi-phase material. PhD-thesis, Technische Universiteit Eindhoven, The Netherlands

28. Lakes RS (1995) Experimental methods for study of Cosserat elastic solids and other generalized elastic continua. In: Mühlhaus HB (ed.), Continuum methods for materials with microstructures, pp 1-25. Wiley, Chichester

29. Laroussi M, Sab K, Alaoui A (2002) Foam mechanics: nonlinear response of an elastic 3D-periodic microstructure. Int J Solids Struct 39:3599-3623

30. Miehe C (1996) Numerical computation of algorithmic (consistent) tangent moduli in large-strain computational inelasticity. Comput Methods Appl Mech Eng 134:223-240

31. Nemat-Nasser S, Hori M (1997) Micromechanics. North-Holland, Amsterdam

32. Nübel V, Düster A, Rank E (2007) An $r p$-adaptive finite element method for elastoplastic problems. Comput Mech 39:557-574
33. Ohno N, Okumura D, Noguchi H (2002) Microscopic symmetric bifurcation condition of cellular solids based on a homogenization theory of finite deformation. J Mech Phys Solids 50:1125-1153

34. Onck PR (2002) Cosserat modeling of cellular solids. C R Mecanique 330:717-722

35. Saiki I, Terada K, Ikeda K, Hori M (2002) Appropriate number of unit cells in a representative volume element for microstructural bifurcation encountered in a multi-scale modeling. Comput Methods Appl Mech Eng 191:2561-2585

36. Schröder J (2000) Homogenisierungsmethoden der nichtlinearen Kontinuumsmechanik unter Beachtung von Stabilitätsproblemen. Habilitationsschrift, Institut für Mechanik (Bauwesen), Number I-7, Universität Stuttgart

37. Szabó BA, Babuška I (1991) Finite element analysis. Wiley, New York

38. Szabó BA, Düster A, Rank E (2004) The p-version of the finite element method. In: Stein E, de Borst R, Hughes TJR (eds) Encyclopedia of computational mechanics, vol 1, chap. 5. Wiley, New York, pp 119-139

39. Szabó BA, Sahrmann GJ (1988) Hierarchic plate and shell models based on p-extension. Int J Numer Methods Eng 26:1855-1881

40. Tekoğlu C (2007) Size effects in cellular solids. PhD thesis, Rijksuniversiteit Groningen

41. Triantafyllidis N, Schraad MW (1998) Onset of failure in aluminum honeycombs under general in-plane loading. J Mech Phys Solids 46:1089-1124

42. Wang Y, Cuitiño AM (2000) Three-dimensional nonlinear opencell foams with large deformations. J Mech Phys Solids 48: 961-988

43. Wriggers P (2001) Nichtlineare Finite-Element-Methoden. Springer, Berlin

44. Yvonnet J, Zahrouni H, Potier-Ferry M (2007) A model reduction method for the post-buckling analysis of cellular microstructures. Comput Methods Appl Mech Eng 197:265-280

45. Zhu HX, Mills NJ, Knott JF (1997) Analysis of the high strain compression of open-cell foams. J Mech Phys Solids 45:1875-1904 